Homology, Homotopy and Applications, vol.14(2), 2012, pp.167-187

\title{
HOMOLOGY DECOMPOSITIONS AND GROUPS INDUCING FUSION SYSTEMS
}

\author{
ASSAF LIBMAN AND NORA SEELIGER \\ (communicated by Jesper Grodal)
}

\begin{abstract}
We relate the construction of groups which realize saturated fusion systems and signaliser functors with homology decompositions of $p$-local finite groups. We prove that the cohomology ring of Robinson's construction is in some precise sense very close to the cohomology ring of the fusion system it realizes.
\end{abstract}

\section{The main results}

The starting point of this paper is the results of Leary-Stancu in [9] and Robinson in [17]. For any saturated fusion system $\mathcal{F}$ on a finite $p$-group $S$ they give recipes to construct groups $\pi_{L S}$ (for Leary-Stancu) and $\pi_{R}$ (for Robinson) which are generally infinite and which contain $S$ as a Sylow $p$-subgroup, see $\S 2.2$, and realize $\mathcal{F}$ in the sense that $\mathcal{F}=\mathcal{F}_{S}(\pi)$. The cohomological properties of the classifying spaces of these constructions were first studied in [19]. Also, for primes $q=3,5 \bmod (8)$, Aschbacher and Chermak constructed in [1] an infinite group $\pi_{A C}$ which contains a Sylow subgroup of $\operatorname{Spin}_{7}\left(q^{n}\right)$ as its Sylow $p$-subgroup and which realizes Solomon's fusion system $\mathcal{F}_{\text {Sol }}\left(q^{n}\right)$, see [10].

The primary goal of this paper is to give a uniform and conceptual treatment for all these constructions. Our approach is geometric rather than algebraic. The main observation in this paper is Theorem 1.1 below which we prove in the end of section 2 . Also, it was observed by Aschbacher that if a saturated fusion system $\mathcal{F}$ has the form $\mathcal{F}_{S}(\pi)$, where $\pi$ is possibly infinite, then a signaliser functor on $\pi$, see $\S 2.2$ for details, gives rise to an associated linking system $\mathcal{L}$. A partial converse to this observation is point 2 of Theorem 1.1.

Theorem 1.1. Fix a p-local finite group $(S, \mathcal{F}, \mathcal{L})$ and let $\pi$ be a group which contains $S$ as a Sylow p-subgroup. Assume that $\mathcal{F} \subseteq \mathcal{F}_{S}(\pi)$ and that there exists a map $f: B \pi \rightarrow|\mathcal{L}|_{p}^{\wedge}$ whose restriction to $B S \subseteq B \pi$ is homotopic to the natural map $\theta: B S \rightarrow|\mathcal{L}|_{p}^{\wedge}$. Then

(1) $\mathcal{F}=\mathcal{F}_{S}(\pi)$

(2) There is a signaliser functor $\Theta$ on $\pi$ which induces $\mathcal{L}$, namely $\mathcal{L}$ is a quotient of the transporter system $\mathcal{T}_{S}^{c}(\pi)$.

Received July 26, 2011, revised July 23, 2012; published on November 26, 2012. 2000 Mathematics Subject Classification: 55R35, 20J99.

Key words and phrases: fusion system, signalizer functor, homology decomposition.

Article available at http://intlpress.com/HHA/v14/n2/a10 and doi:10.4310/HHA.2012.v14.n2.a10

Copyright (C) 2012, International Press. Permission to copy for private use granted. 
(3) $H^{*}\left(|\mathcal{L}| ; \mathbb{F}_{p}\right)$ is a retract of $H^{*}\left(B \pi ; \mathbb{F}_{p}\right)$ in the category of $\mathbb{F}_{p}$-algebras. It is equal to the image of $H^{*}\left(\pi ; \mathbb{F}_{p}\right) \stackrel{\text { res }}{\longrightarrow} H^{*}\left(S ; \mathbb{F}_{p}\right)$.

The point of Propositions 4.1, 4.5 and 4.9 below is that the groups $\pi_{L S}, \pi_{R}$ and $\pi_{A C}$ satisfy the hypotheses of this theorem, hence recovering the results of $[\mathbf{9}, \mathbf{1 7}]$ and $[\mathbf{1}]$; See Remarks 1.3-1.6 below. The conceptual reason that Theorem 1.1 applies to these groups, which is the main message of this paper, is that all these groups are built from different homology decompositions which express $|\mathcal{L}|$ as the homotopy colimit of a diagram of classifying spaces of finite groups. By taking homotopy colimits of carefully chosen subdiagrams we obtain classifying spaces $B \pi$ together with maps $B \pi \rightarrow|\mathcal{L}|_{p}^{\wedge}$, where $\pi$ induces the fusion system $\mathcal{F}$. The groups $\pi_{L S}$ are built in $\S 4.2$ from the subgroup decomposition $[\mathbf{4}, \S 2]$. The groups $\pi_{R}$ are built in $\S 4.1$ from the normalizer decomposition with respect to the collection of the $\mathcal{F}$-centric subgroups [12]. The groups $\pi_{A C}$ are related to the normalizer decomposition with respect to the collection of the elementary abelian subgroup of $\mathcal{F}_{\text {Sol }}\left(q^{n}\right)$ as we explain in $\S 4.3$.

A consequence of Theorem 1.1(2) is the following result whose proof is given in $\S \S 4.1$ and $\S \S 4.2$.

Corollary 1.2. Fix a saturated fusion system $\mathcal{F}$ on $S$ and let $\pi$ be either Robinson's group $\pi_{R}$ or Leary-Stancu's group $\pi_{L S}$ so that $\mathcal{F}=\mathcal{F}_{S}(\pi)$. Then the following are equivalent.

(1) $\mathcal{F}$ has an associated linking system $\mathcal{L} .^{1}$

(2) There exists a signaliser functor on $\pi$ which induces $\mathcal{L}$.

Remark 1.3. We explain in Remark 4.2 below, see also Proposition 2.2, that the existence of an associated linking system is not essential to deduce point (1) of Theorem 1.1 for arbitrary saturated fusion systems. Thus, even though Theorem 1.1 is not quite stated in this way, our results are as strong as those of [9] and [17] which apply to arbitrary saturated fusion systems.

Remark 1.4. The group $\pi_{R}$ that we construct in $\S 4.1$ is, in fact, a factor group of Robinson's original construction [17]. See Remark 4.3 below. Our construction of $\pi_{R}$ is inspired by the results of the first author and Antonio Viruel in [13].

Remark 1.5. The construction of $\pi_{A C}$ in [1] requires $q \equiv 3$ or $5 \bmod (8)$. This is particularly important for the construction of the signaliser functors. The group $\pi_{A C}$ that we construct in $\S 4.3$ is, in fact, a variation of Aschbacher-Chermak's original construction. Our group which is motivated by the geometric approach, requires no restriction on $q \bmod (8)$, see Remark 4.10. On the down side, we cannot give an explicit description of the signaliser functor as Aschbacher and Chermak do.

Remark 1.6. Finally, point (3) of Theorem 1.1 applies to $\pi_{L S}, \pi_{R}$ and $\pi_{A C}$ and, as far as we know, this cohomological information is new.

From the cohomological point of view, the groups $\pi_{R}$ and $\pi_{A C}$ are very close to their "target" namely the ring $\mathcal{H}^{*}(\mathcal{F})$ of the stable elements, see [4, Theorem B], in

\footnotetext{
${ }^{1}$ Chermak and Oliver have recently announced the result that every saturated fusion system admits
} a unique associated centric linking system. 
a sense made precise in the next result which is proven in $\S \S 4.1,4.3$. Recall that a space $X$ is called $p$-good in the sense of Bousfield-Kan, [6, I. $\S 5]$, if the natural map $X \rightarrow X_{p}^{\wedge}$ induces an isomorphism in mod- $p$ homology.

Proposition 1.7. Let $\pi$ be either the group $\pi_{R}$ associated to a saturated fusion system $\mathcal{F}$ (see §4.1) or the group $\pi_{A C}$ if $\mathcal{F}=\mathcal{F}_{\text {Sol }}\left(q^{n}\right)$ and $p=2$ (see §4.3). Then

(1) $B \pi$ is $p$-good in the sense of $[\boldsymbol{6}, I . \S 5]$.

(2) In the kernel of $H^{*}\left(B \pi ; \mathbb{F}_{p}\right) \stackrel{B i^{*}}{\longrightarrow} H^{*}\left(B S ; \mathbb{F}_{p}\right)$ the product of any two elements is zero.

\section{Saturated fusion systems and $p$-local finite groups}

In this section we will very briefly recall the notion of $p$-local finite groups and of transporter systems. We will take the approach of Broto Levi and Oliver in $[4]$ and Oliver-Ventura [14, $\S \S 2,3]$. Also see [5]. Fusion systems were first defined by Puig, e.g., $[\mathbf{1 5}]$.

\subsection{Fusion systems}

A fusion system on a finite $p$-group $S$ is a small category $\mathcal{F}$ whose object set is the set of subgroups of $S$. Morphism sets $\mathcal{F}(P, Q)$ are group monomorphisms which include the set $\operatorname{Hom}_{S}(P, Q)$ of conjugations by elements of $S$. In addition, every morphism $P \rightarrow Q$ in $\mathcal{F}$ is the composition of an isomorphism $P \rightarrow P^{\prime}$ in $\mathcal{F}$, which is necessarily a group isomorphism, followed by an inclusion $P^{\prime} \leqslant Q$. From the definitions, every endomorphism in $\mathcal{F}$ is an automorphism.

Subgroups $P, P^{\prime} \leqslant S$ are called $\mathcal{F}$-conjugate if they are isomorphic as objects of $\mathcal{F}$. A subgroup $P \leqslant S$ is called fully centralized if $P$ has the largest $S$-centralizer among all the subgroups in its $\mathcal{F}$-conjugacy class. Similarly, $P$ is fully normalized if its $S$-normalizer has the largest possible order among all the groups in its $\mathcal{F}$ conjugacy class. We say that $\mathcal{F}$ is saturated if the following two conditions hold. First, every fully normalized subgroup $P \leqslant S$ is fully centralized and in this case $\operatorname{Aut}_{S}(P)=N_{S}(P) / C_{S}(P)$ is a Sylow $p$-subgroup of $\operatorname{Aut}_{\mathcal{F}}(P)$. Second, if $\varphi \in \mathcal{F}(P, S)$ is such that $\varphi(P)$ is fully centralized then $\varphi$ extends to a morphism $\tilde{\varphi} \in \mathcal{F}\left(N_{\varphi}, S\right)$, where $N_{\varphi} \leqslant N_{S}(P)$ consists of the elements $g$ such that $\varphi \circ c_{g} \circ \varphi^{-1} \in \operatorname{Aut}_{S}(\varphi(P))$, where $c_{g}$ is the inner automorphism $x \mapsto g x g^{-1}$ of $S$.

A subgroup $P \leqslant S$ is called $\mathcal{F}$-centric if $P$ and all its $\mathcal{F}$-conjugates contain their $S$-centralizer. We let $\mathcal{F}^{c}$ denote the full subcategory of $\mathcal{F}$ on the $\mathcal{F}$-centric objects. A subgroup $P \leqslant S$ is called $\mathcal{F}$-radical if $\operatorname{Inn}(P)$ is the maximal normal $p$-subgroup of $\operatorname{Aut}_{\mathcal{F}}(P)$. Alperin's fusion theorem [4, Theorem A.10] says that any saturated fusion system $\mathcal{F}$ is generated by the $\mathcal{F}$-automorphism groups of its $\mathcal{F}$-centric $\mathcal{F}$-radical subgroups. Namely, every morphism in $\mathcal{F}$ is obtained by composition of restrictions of $\mathcal{F}$-automorphisms of these groups.

An important source of saturated fusion systems are finite groups. If $S$ is a Sylow $p$-subgroup of a finite group $G$, one forms the category $\mathcal{F}_{S}(G)$ whose objects are the subgroups of $S$ and whose morphisms are the group monomorphisms $c_{g}: P \rightarrow Q$ of the form $x \mapsto g x g^{-1}$ for some $g \in G$. 


\subsection{Transporter systems, linking systems and signaliser functors}

A finite $p$-subgroup $S$ of a group $\pi$, possibly infinite, is called a Sylow p-subgroup if any finite $p$-subgroup $Q \leqslant \pi$ is conjugate to a subgroup of $S$.

Define $\mathcal{F}_{S}(\pi)$ where $S \leqslant \pi$ is a Sylow $p$-subgroup as we did above for finite groups; Up to an isomorphism of fusion systems, the choice of $S$ is immaterial.

The objects of the transporter system $\mathcal{T}_{S}(\pi)$ are the subgroups of $S$; its morphism sets are

$$
\mathcal{T}_{S}(\pi)(P, Q)=N_{\pi}(P, Q)=\left\{g \in \pi: g P g^{-1} \leqslant Q\right\} .
$$

There is an obvious surjective functor $\mathcal{T}_{S}(\pi) \rightarrow \mathcal{F}_{S}(\pi)$ which is the identity on objects and is the quotient by the action of $C_{\pi}(P)$ on morphism sets. We denote by $\mathcal{T}_{S}^{c}(\pi)$ the full subcategory whose objects are the $\mathcal{F}$-centric subgroups of $S$ for some fusion system $\mathcal{F}$ on $S$ which will be understood from the context.

An associated centric linking system $\mathcal{L}$ to a saturated fusion system $\mathcal{F}$ on $S$ is a category whose objects are the $\mathcal{F}$-centric subgroups. It is equipped with a surjective functor $\pi: \mathcal{L} \rightarrow \mathcal{F}^{c}$ and an injective functor $\delta: \mathcal{T}_{S}^{c}(S) \rightarrow \mathcal{L}$, both induce the identity on object sets. In addition the following three conditions must be satisfied. First, the image of $Z(P)$ under $\delta: N_{S}(P) \rightarrow \operatorname{Aut}_{\mathcal{L}}(P)$ acts freely on $\mathcal{L}(P, Q)$, and $\mathcal{F}(P, Q)$ is isomorphic to the orbit set via $\pi$. Second, for any $g \in N_{S}(P, Q)$ we have $\pi(\delta(g))=c_{g}$. Third, for any $f \in \mathcal{L}(P, Q)$ and any $g \in P \leqslant N_{S}(P)=\operatorname{Aut}_{\mathcal{T}_{S}^{c}(S)}(P)$ we have $\delta(\pi(f)(g)) \circ f=f \circ \delta(g)$ in $\mathcal{L}$.

A $p$-local finite group is a triple $(S, \mathcal{F}, \mathcal{L})$ of a saturated fusion system on $S$ together with an associated linking system.

Now suppose that $\mathcal{F}=\mathcal{F}_{S}(\pi)$ for a group $\pi$ which contains $S$ as a Sylow $p$ subgroup. A signaliser functor on $\pi$ is an assignment $P \mapsto \Theta(P)$ for every $\mathcal{F}$-centric $P \leqslant S$ such that $\Theta(P)$ is a complement of $Z(P)$ in $C_{\pi}(P)$ and such that if $g P g^{-1} \leqslant Q$ then $\Theta(Q) \leqslant g \Theta(P) g^{-1}$. Indeed, $\Theta$ is a contravariant functor from $\mathcal{T}_{S}^{c}(\pi)$ to the category of groups. A signaliser functor $\Theta$, if it exists, gives rise to a centric linking system $\mathcal{L}=\mathcal{L}_{\Theta}$, where $\mathcal{L}(P, Q)=N_{\pi}(P, Q) / \Theta(P)$. See [1] and also [13, Section 3] for more details.

\subsection{Homotopy theoretic constructions}

Fix a finite $p$-group $S$ and a map of topological spaces $f: B S \rightarrow X$. By [4, Definition 7.1] $f$ gives rise to a fusion system $\mathcal{F}_{S}(f)$ on $S$ whose objects are the subgroups of $S$ and a group monomorphism $\varphi: P \rightarrow Q$ is a morphism in $\mathcal{F}_{S}(f)$ if and only if $B P \stackrel{B \varphi}{\longrightarrow} B S \stackrel{f}{\rightarrow} X$ is homotopic to $B P \stackrel{B i}{\rightarrow} B S \stackrel{f}{\rightarrow} X$. It follows directly from the definitions that any map $g: X \rightarrow Y$ of topological spaces induces an inclusion of fusion systems (not saturated in general)

$$
\mathcal{F}_{S}(f) \subseteq \mathcal{F}_{S}(g \circ f) .
$$

The category $\mathcal{L}_{S}(f)$, see [4, Definition 7.2], has the same object set as $\mathcal{F}=\mathcal{F}_{S}(f)$. The morphisms $P \rightarrow Q$ in $\mathcal{L}_{S}(f)$ are pairs $(\varphi,[H])$, where $\varphi \in \mathcal{F}(P, Q)$ and $[H]$ is the homotopy class of a path $H$ in $\operatorname{map}(B P, X)$ from $B P \stackrel{B \varphi}{\longrightarrow} B S \stackrel{f}{\rightarrow} X$ to $B P \stackrel{B i}{\longrightarrow}$ $B S \stackrel{f}{\rightarrow} X$. There is a surjective functor $\pi: \mathcal{L}_{S}(f) \rightarrow \mathcal{F}_{S}(f)$ which "forgets" $[H]$. 
A map $g: X \rightarrow Y$ gives rise, by composition, to a functor

$$
g_{*}: \mathcal{L}_{S}(f) \rightarrow \mathcal{L}_{S}(g \circ f)
$$

over the projection functors to $\mathcal{F}_{S}(f) \subseteq \mathcal{F}_{S}(g \circ f)$, i.e., $\pi \circ g_{*}=\operatorname{incl}_{\mathcal{F}_{S}(f)}^{\mathcal{F}_{S}(g f)} \circ \pi$.

Define $\mathcal{L}_{S}^{c}(f)$ as the full subcategory of $\mathcal{L}_{S}(f)$ on the $\mathcal{F}_{S}(f)$-centric objects. If $g: X \rightarrow Y$ is a map then $\mathcal{F}_{S}(f) \subseteq \mathcal{F}_{S}(g \circ f)$ so every $\mathcal{F}_{S}(g \circ f)$-centric subgroup of $S$ is also $\mathcal{F}_{S}(f)$-centric and we obtain a functor

$$
g_{*}: \mathcal{L}_{S}^{c}(f) \rightarrow \mathcal{L}_{S}^{c}(g \circ f)
$$

defined on the $\mathcal{F}_{S}(g \circ f)$-centric objects of $\mathcal{L}_{S}^{c}(f)$.

\subsection{Classifying spaces}

The classifying space of a $p$-local finite group is $|\mathcal{L}|_{p}^{\wedge}$. The inclusion $\delta: S \rightarrow \operatorname{Aut}_{\mathcal{L}}(S)$ thought of as a full subcategory of $\mathcal{L}$ gives rise to the map

$$
\theta: B S \rightarrow|\mathcal{L}|_{p}^{\wedge}
$$

which will play a fundamental role in this paper. One of the key features of the map $\theta$ is $[4$, Proposition 7.3]

$$
\mathcal{F}=\mathcal{F}_{S}(\theta) \text { and } \mathcal{L}=\mathcal{L}_{S}^{c}(\theta) .
$$

For any group $G$ let $\mathcal{B} G$ denote the category with one object $o_{G}$ and $G$ as its automorphism set. A homomorphism $\varphi: G^{\prime} \rightarrow G$ induces a functor $\mathcal{B} \varphi: \mathcal{B} G^{\prime} \rightarrow \mathcal{B} G$. It is well known that if $H \leqslant G$ then the functor $\mathcal{B} C_{G}(H) \times \mathcal{B} H \stackrel{(x, y) \mapsto x y}{\longrightarrow} \mathcal{B} G$ gives rise, upon realization and adjunction, to a homotopy equivalence $B C_{G}(H) \stackrel{\simeq}{\rightarrow} \operatorname{map}(B H, B H)_{B i}$, where the space on the right is the path component of the inclusion $B H \stackrel{B i}{\longrightarrow} B G$.

Proposition 2.1. Fix an inclusion $S \leqslant \pi$ of a Sylow p-subgroup in a discrete group $\pi$, and consider $B i: B S \rightarrow B \pi$. Then $\mathcal{F}_{S}(B i)=\mathcal{F}_{S}(\pi)$ and $\mathcal{L}_{S}(B i)=\mathcal{T}_{S}(\pi)$.

Proof. The first isomorphism is clear because $[B P, B \pi]=\operatorname{Hom}(P, \pi) / \operatorname{Inn}(\pi)$. Define a functor $\Phi: \mathcal{T}_{S}(\pi) \rightarrow \mathcal{L}_{S}(B i)$ as follows. It is the identity on objects and for a morphism $g \in N_{\pi}(P, Q)$, where $P, Q \leqslant S$, consider $c_{g}: P \rightarrow Q$ and the natural transformation $g: \mathcal{B} c_{g} \rightarrow \mathcal{B} i_{P}^{\pi}$ between the functors $c_{g}, i_{P}^{\pi}: \mathcal{B P} \rightarrow \mathcal{B} \pi$ given by $o_{\pi} \stackrel{g}{\rightarrow} o_{\pi}$. This natural transformation gives rise to a homotopy $I \times B P \rightarrow B \pi$ from $B c_{g}$ and $B i_{P}^{\pi}$, hence to a path $H$ in $\operatorname{map}(B P, B \pi)$ from $B c_{g}$ to $B i_{P}^{\pi}$. Define $\Phi(g)=\left(c_{g},[H]\right)$.

The functoriality of $\Phi$ is easy to check. It is bijective because $\Phi$ maps the coset $C_{\pi}(P) g \leqslant N_{\pi}(P, Q)$ bijectively onto $\pi_{1}^{\#}\left(\operatorname{map}(B P, B \pi) ; B c_{g}, B i_{P}^{\pi}\right) \cong \pi_{1} B C_{\pi}(P)$ via $\mathcal{B} C_{\pi}(P) \times \mathcal{B} P \rightarrow \mathcal{B} \pi$. Here $\pi_{1}^{\#}\left(X ; x_{0}, x_{1}\right)$ is the set of homotopy classes of paths from $x_{0}$ to $x_{1}$ in a topological space $X$. The homotopy equivalence

$$
\operatorname{map}(B H, B G) \simeq \coprod_{\varphi \in \operatorname{Hom}(H, G) / \operatorname{Inn}(G)} B C_{G}(\varphi(H)),
$$

where $H$ and $G$ are discrete groups is classical. It follows from standard facts on covering spaces applied to the evaluation fibre sequence

$$
\operatorname{map}_{*}(B H, B G) \rightarrow \operatorname{map}(B H, B G) \stackrel{\text { ev }}{\longrightarrow} B G
$$

exploiting the fact that $\operatorname{map}_{*}(B H, B G)$ is homotopically discrete by induction on 
the skeleta of $B H$ and that its set of components is $\operatorname{Hom}(H, G)$ by $[\mathbf{2 0}$, Theorem 9 , Chapter 8.1] with $G$ acting via $\operatorname{Inn}(G)$.

\subsection{The subgroup decomposition}

Recall that the orbit category $\mathcal{O}=\mathcal{O}(\mathcal{F})$ of a fusion system $\mathcal{F}$ has the same object set as $\mathcal{F}$ and $\mathcal{O}(P, Q)=\mathcal{F}(P, Q) / \operatorname{Inn}(Q)$. See [4, Definition 2.1]. Let $\mathcal{O}\left(\mathcal{F}^{c}\right)$ denote the full subcategory on the $\mathcal{F}$-centric objects.

Fix a $p$-local finite group $(S, \mathcal{F}, \mathcal{L})$. By $[\mathbf{4}$, Proposition 2.2] there is a functor called the subgroup decomposition

$$
\beta: \mathcal{O}\left(\mathcal{F}^{c}\right) \rightarrow \text { Top }
$$

which as a functor into hoTop - the homotopy category of spaces - is isomorphic to the functor $P \mapsto B P$. Moreover, there is a homotopy equivalence

$$
\operatorname{hocolim}_{\mathcal{O}\left(\mathcal{F}^{c}\right)} \beta \stackrel{g}{\rightarrow}|\mathcal{L}|
$$

whose restriction to $\beta(S) \simeq B S$ is homotopic to the map $B S \rightarrow|\mathcal{L}|$ induced by the inclusion $S \leqslant \operatorname{Aut}_{\mathcal{L}}(S)$. Hence $\left(\left.g\right|_{B S}\right)_{p}^{\wedge}$ is homotopic to $\theta$ in display (1) in $\S 2.4$.

\subsection{The normalizer decomposition}

Let $\mathcal{R}$ be a set of $\mathcal{F}$-centric subgroups of $S$ which is closed under $\mathcal{F}$-conjugation. The subdivision category $\bar{S}(\mathcal{R})$ is the following poset; see [12] for more details, where it is denoted $\bar{s} d(\mathcal{R})$. The objects of $\bar{S}(\mathcal{R})$ are the $\mathcal{F}$-conjugacy classes $\left[P_{\bullet}\right]$ of chains of inclusions $P_{0} \lesseqgtr \ldots \leq P_{k}$ of subgroups in $\mathcal{R}$ called " $k$-simplices" $(k \geqslant 0)$. We say that $P_{\bullet}$ is $\mathcal{F}$-conjugate to $P_{\bullet}^{\prime}=P_{0}^{\prime} \lesseqgtr \ldots \lesseqgtr P_{k}^{\prime}$ if there is an isomorphism $P_{k} \rightarrow P_{k}^{\prime}$ in $\mathcal{F}$ which carries $P_{i}$ isomorphically onto $P_{i}^{\prime}$ for all $i$. There is a unique morphism $\left[P_{\bullet}\right] \rightarrow\left[Q_{\bullet}\right]$ in $\bar{S}(\mathcal{R})$ if $Q_{\bullet}$ is $\mathcal{F}$-conjugate to a sub-sequence (a "subsimplex") of $P_{\bullet}$.

Let $\operatorname{Aut}_{\mathcal{F}}\left(P_{\bullet}\right)$ be the subgroup of $\operatorname{Aut}_{\mathcal{F}}\left(P_{k}\right)$ which leaves $P_{0}, \ldots, P_{k}$ invariant. Let $\operatorname{Aut}_{\mathcal{L}}\left(P_{\bullet}\right)$ be its preimage in $\operatorname{Aut}_{\mathcal{L}}\left(P_{k}\right)$. Suppose that $Q_{\bullet}=Q_{0} \lesseqgtr \ldots \lessgtr Q_{m}$ is a sub-simplex of $P_{\bullet}$, where $Q_{m}=P_{i}$ for some $i$. Consider $e \in N_{\pi}\left(P_{i}, P_{k}\right)$ and set $\hat{e}=\delta(e) \in \mathcal{L}\left(P_{i}, P_{k}\right)$. For every $\varphi \in \operatorname{Aut}_{\mathcal{L}}\left(P_{\bullet}\right)$ we have $\pi(\varphi) \in \operatorname{Aut}_{\mathcal{F}}\left(P_{\bullet}\right)$ and therefore $\left.\pi(\varphi)\right|_{P_{i}} \in \operatorname{Aut}_{\mathcal{F}}\left(Q_{\bullet}\right)$. It follows from [4, Lemma 1.10(a)] and the fact that $\hat{e}$ is both an epimorphism and a monomorphism in $\mathcal{L}$, see [2, Corollary 3.10] that there exists a unique $\varphi^{\prime} \in \operatorname{Aut}_{\mathcal{L}}\left(Q_{\bullet}\right)$ such that $\varphi \circ \hat{e}=\hat{e} \circ \varphi^{\prime}$. This sets up a canonical group monomorphism $\operatorname{Aut}_{\mathcal{L}}\left(P_{\bullet}\right) \hookrightarrow \operatorname{Aut}_{\mathcal{L}}\left(Q_{\bullet}\right)$. See $[\mathbf{1 2}]$ for more details. We obtain a functor $\left[P_{\bullet}\right] \mapsto B \operatorname{Aut}_{\mathcal{L}}\left(P_{\bullet}\right)$ into the homotopy category of spaces (here we use the fact that the self-map that an inner automorphism of a group induces on its classifying space is homotopic to the identity).

The normalizer decomposition with respect to the collection $\mathcal{R}$ of $\mathcal{F}$-centric subgroups, see $[\mathbf{1 2}$, Theorem A], is a functor

$$
\delta: \bar{S}(\mathcal{R}) \rightarrow \text { Top }
$$

which as a functor into hoTop is isomorphic to the functor $\left[P_{\bullet}\right] \mapsto B \operatorname{Aut}_{\mathcal{L}}\left(P_{\bullet}\right)$. In addition there is a map

$$
\operatorname{hocolim}_{\bar{S}(\mathcal{R})} \delta \stackrel{g}{\rightarrow}|\mathcal{L}|
$$

whose restriction to $\delta\left(\left[P_{\bullet}\right]\right)$ is induced, upon taking nerves, from the inclusion of categories $\mathcal{B A u t}_{\mathcal{L}}\left(P_{\bullet}\right) \subseteq \mathcal{L}$. Hence, the restriction of $\operatorname{hocolim}_{\bar{S}(\mathcal{R})} \delta \simeq|\mathcal{L}| \rightarrow|\mathcal{L}|_{p}^{\wedge}$ to 
$B S \leqslant B \operatorname{Aut}_{\mathcal{L}}(S)$ is homotopic to $\theta$ in display $(1)$ in $\S 2.4$. If $\mathcal{R}$ contains all the $\mathcal{F}$-centric $\mathcal{F}$-radical subgroups of $S$ then $g$ is a homotopy equivalence by [12, Theorem A].

Proof of Theorem 1.1(1). Let $B i: B S \rightarrow B \pi$ denote the inclusion. By Proposition 2.1 the map $f$ induces an inclusion

$$
\mathcal{F}_{S}(\pi)=\mathcal{F}_{S}(B i) \subseteq \mathcal{F}_{S}(f \circ B i)=\mathcal{F}_{S}(\theta)=\mathcal{F} .
$$

By hypothesis $\mathcal{F} \subseteq \mathcal{F}_{S}(\pi)$ and therefore equality holds.

(2) We deduce from Proposition 2.1 and from $\S 2.3$ that $\mathcal{L}_{S}^{c}(B i)=\mathcal{T}_{S}^{c}(\pi)$, that $\mathcal{L}_{S}^{c}(f \circ B i)=\mathcal{L}$ because $f \circ B i \simeq \theta$, and that $f$ induces a functor $\rho: \mathcal{T}_{S}^{c}(\pi) \rightarrow \mathcal{L}$ such that the following diagram commutes.

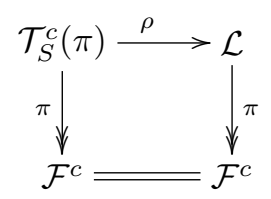

We will now prove, and this is the key observation, that $\rho$ is surjective.

Set $\mathcal{T}=\mathcal{T}_{S}^{c}(\pi)$. Since $Z(P) \leqslant \operatorname{Aut}_{\mathcal{L}}(P)$ acts on $\mathcal{L}(P, Q)$ with orbit set $\mathcal{F}(P, Q)$ and since the functors $\pi$ in the square above are surjective, it suffices to prove that $Z(P)$ is in the image of $\rho_{P}: \operatorname{Aut}_{\mathcal{T}}(P) \rightarrow \operatorname{Aut}_{\mathcal{L}}(P)$ for any $\mathcal{F}$-centric $P \leqslant S$. To do this, consider the inclusion $B i_{P}^{S}: B P \rightarrow B S$. The composition

$$
\operatorname{map}(B P, B S)_{B i_{P}^{S}} \stackrel{B i_{*}}{\longrightarrow} \operatorname{map}(B P, B \pi)_{B i_{P}^{\pi}} \stackrel{f_{*}}{\longrightarrow} \operatorname{map}\left(B P,|\mathcal{L}|_{p}^{\wedge}\right)_{f \circ B i_{P}^{\pi}}
$$

is a homotopy equivalence because $P$ is $\mathcal{F}$-centric so the first space is homotopy equivalent to $B C_{S}(P)=B Z(P)$ and because the third space is homotopy equivalent to $B Z(P)$ by $[4$, Theorem 4.4(c)] since $B i \circ f \simeq \theta$. We see from the description of $\mathcal{L}_{S}(-)$ in $\S 2.3$, that $\rho_{P}$ carries $Z(P) \leqslant N_{\pi}(P)$ isomorphically onto $Z(P) \leqslant \operatorname{Aut}_{\mathcal{L}}(P)$.

We will now define the signaliser functor $\Theta$. For every $\mathcal{F}$-centric $P \leqslant S$ set

$$
\Theta(P) \stackrel{\text { def }}{=} \operatorname{Ker}\left(\operatorname{Aut}_{\mathcal{T}}(P) \rightarrow \operatorname{Aut}_{\mathcal{L}}(P)\right) .
$$

Now, $\operatorname{Aut}_{\mathcal{T}}(P)=N_{\pi}(P)$ so we obtain a commutative diagram with exact rows

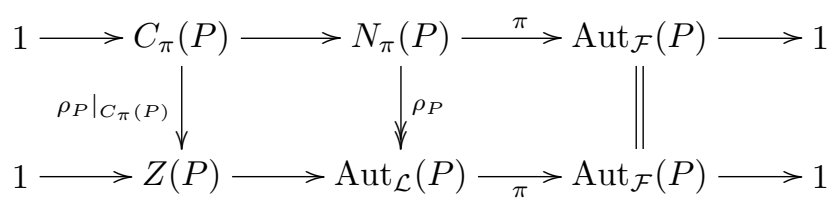

which implies that $\Theta(P) \leqslant C_{\pi}(P)$ and that it is a complement of $Z(P)$ because we have seen that $\rho_{P}(Z(P))=Z(P)$. For $g \in \pi$ such that $g P g^{-1} \leqslant S$ we obtain a commutative diagram

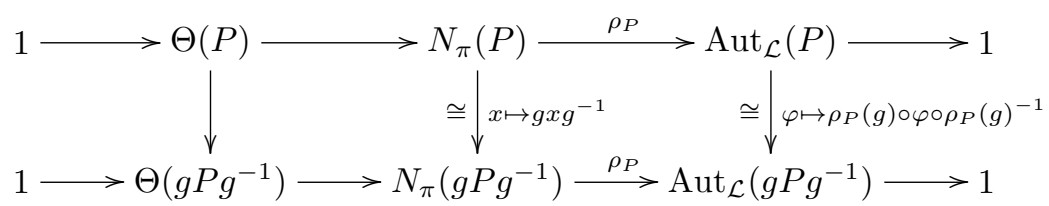

which shows that $g \Theta(P) g^{-1}=\Theta\left(g P g^{-1}\right)$. 
Now suppose that $P \leqslant Q$ and set $N=N_{\pi}(P) \cap N_{\pi}(Q)$. Set $\hat{e}=\delta(e) \in \mathcal{L}(P, Q)$, where $e \in N_{S}(P, Q)$ is the identity element. Note that $\rho_{P, Q}(e)=\hat{e} \circ \delta_{P}(z)$ for some $z \in Z(P)$ because $\hat{e}$ and $\rho_{P, Q}(e)$ project to the inclusion $P \leqslant Q$ in $\mathcal{F}$. Let $\operatorname{Aut}_{\mathcal{L}}\left(Q, \downarrow_{P}\right)$ be the subgroup of $\operatorname{Aut}_{\mathcal{L}}(Q)$ of the automorphisms $\varphi$ which restrict to an automorphism of $P$ in the sense that there exists $\varphi^{\prime} \in \operatorname{Aut}_{\mathcal{L}}(P)$ such that $\varphi \circ \hat{e}=\hat{e} \circ \varphi^{\prime}$. Note that $\varphi^{\prime}$ is unique because $\hat{e}$ is a monomorphism in $\mathcal{L}$ by [2, Corollary 3.10]. Similarly, let $\operatorname{Aut}_{\mathcal{L}}\left(P, \uparrow^{Q}\right)$ denote the subgroup of $\operatorname{Aut}_{\mathcal{L}}(P)$ of the automorphisms $\varphi^{\prime}$ which extend to an automorphism of $Q$ in the sense that there exists $\varphi \in \operatorname{Aut}_{\mathcal{L}}(Q)$ such that $\varphi \circ \hat{e}=\hat{e} \circ \varphi^{\prime}$. Note that $\varphi$ is unique because $\hat{e}$ is an epimorphism in $\mathcal{L}$ by $[\mathbf{2}$, Corollary 3.10]. Thus $\varphi \mapsto \varphi^{\prime}$ sets up an isomorphism $\operatorname{Aut}_{\mathcal{L}}\left(Q, \downarrow_{P}\right) \rightarrow \operatorname{Aut}_{\mathcal{L}}(P, \uparrow Q)$. Moreover, if $\varphi=\rho_{Q}(g)$ for some $g \in N_{\pi}(Q)$, then $g \in N$ and $\varphi^{\prime}=\delta_{P}(z) \circ \rho_{P}(g) \circ \delta_{P}(z)^{-1}$ for the element $z \in Z(P)$ described above.

Clearly, the preimage under $\rho$ of $\operatorname{Aut}_{\mathcal{L}}\left(Q, \downarrow_{P}\right)$ in $\operatorname{Aut}_{\mathcal{T}}(Q)=N_{\pi}(Q)$ is $N$ as is the preimage of $\operatorname{Aut}_{\mathcal{L}}(P, \uparrow Q)$ in $\operatorname{Aut}_{\mathcal{T}}(P)=N_{\pi}(P)$. Also, $C_{\pi}(Q) \leqslant N$. We obtain

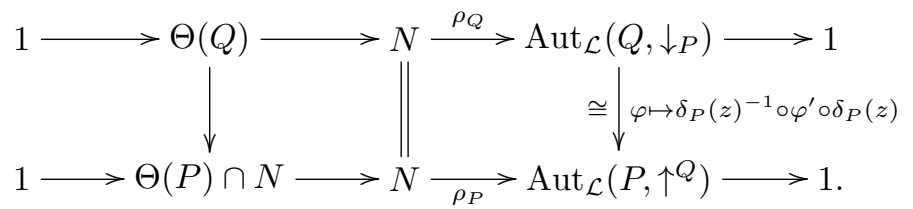

We deduce that $\Theta(Q)=\Theta(P) \cap N \leqslant \Theta(P)$, hence $\Theta$ is a signaliser functor.

(3) Fix some $\varphi \in \mathcal{F}(P, Q)$. There is some $g \in \pi$ such that $\varphi$ is the restriction of $c_{g} \in \operatorname{Inn}(\pi)$ to $P$ because $\mathcal{F}=\mathcal{F}_{S}(\pi)$. Since $B c_{g}$ is homotopic to the identity on $B \pi$, the following square commutes up to homotopy

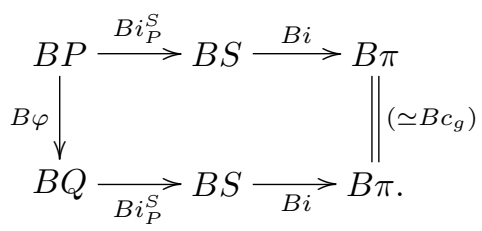

Applying $H^{*}\left(-; \mathbb{F}_{p}\right)$ to this diagram for every $\varphi \in \mathcal{F}^{c}$ shows that the image of the ring homomorphism $H^{*}\left(B \pi ; \mathbb{F}_{p}\right) \stackrel{B i^{*}}{\longrightarrow} H^{*}\left(B S ; \mathbb{F}_{p}\right)$ is contained in the ring of stable elements $H^{*}\left(\mathcal{F}^{c} ; \mathbb{F}_{p}\right)$, namely the subring of all the classes $\alpha \in H^{*}\left(S ; \mathbb{F}_{p}\right)$ such that $\varphi^{*}\left(\left.\alpha\right|_{Q}\right)=\left.\alpha\right|_{P}$ for all $\varphi \in \mathcal{F}^{c}(P, Q)$.

Now we consider $B S \stackrel{B i}{\longrightarrow} B \pi \stackrel{f}{\rightarrow}|\mathcal{L}|_{p}^{\wedge}$ which is homotopic to $\theta$. By [4, Theorem 5.8] $\theta^{*}$ is an isomorphism of $H^{*}\left(|\mathcal{L}|_{p}^{\wedge} ; \mathbb{F}_{p}\right)$ onto $H^{*}\left(\mathcal{F}^{c}\right) \leqslant H^{*}\left(B S ; \mathbb{F}_{p}\right)$. But $\theta^{*}=(B i)^{*} \circ$

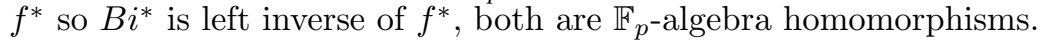

Ragnarsson defined in [16] a classifying spectrum $\mathbb{B} \mathcal{F}$ for any saturated fusion system $\mathcal{F}$. It is equipped with a structure map $\sigma_{\mathcal{F}}: \mathbb{B} S \rightarrow \mathbb{B} \mathcal{F}$, where $\mathbb{B} S=\Sigma^{\infty} B S$. In the presence of a linking system, $\mathbb{B} \mathcal{F} \simeq \Sigma^{\infty}|\mathcal{L}|_{p}^{\wedge}$ and $\sigma_{\mathcal{F}}=\Sigma^{\infty} \theta$, see display (1). He showed that the assignment of $\mathcal{F} \mapsto \mathbb{B} \mathcal{F}$ is a functor from the category of fusion systems to the homotopy category of spectra. In addition he showed that $\mathcal{F}$ can be recovered from $\left(\mathbb{B} \mathcal{F}, \sigma_{\mathcal{F}}\right)$ in the sense that the morphisms in $\mathcal{F}$ are exactly the group monomorphisms $\varphi: P \rightarrow Q$ such that $\mathbb{B} P \stackrel{\mathbb{B} \varphi}{\longrightarrow} \mathbb{B} S \stackrel{\sigma_{\mathcal{F}}}{\longrightarrow} \mathbb{B} \mathcal{F}$ is homotopic to $\mathbb{B} P \stackrel{\mathbb{B} i}{\longrightarrow}$ 
$\mathbb{B} S \stackrel{\sigma_{\mathcal{F}}}{\longrightarrow} \mathbb{B} \mathcal{F}$, where $\mathbb{B} P=\Sigma^{\infty} B P$. Compare this with the construction in $\S 2.3$. Here is a variant of Theorem 1.1(1).

Proposition 2.2. Suppose $\mathcal{F}$ is a saturated fusion system on $S$ and that $S$ is a Sylow p-subgroup of some group $\pi$. Assume that there exists a map $f: B \pi \rightarrow \Omega^{\infty} \mathbb{B} \mathcal{F}$ whose restriction to $B S$ is homotopic to the right adjoint $\sigma_{\mathcal{F}}^{\#}: B S \rightarrow \Omega^{\infty} \mathbb{B} \mathcal{F}$ of $\sigma_{\mathcal{F}}: \mathbb{B} S \rightarrow$ $\mathbb{B} \mathcal{F}$. Then $\mathcal{F}_{S}(\pi) \subseteq \mathcal{F}$.

Proof. By Proposition 2.1 and the constructions in $\S 2.3$, it only remains to prove that $\mathcal{F}=\mathcal{F}_{S}\left(\sigma_{\mathcal{F}}^{\#}\right)$. This is straightforward from the definition of $\mathcal{F}_{S}\left(\sigma_{\mathcal{F}}^{\#}\right)$ and the adjunction $[\mathbb{B} P, \mathbb{B} \mathcal{F}] \cong\left[\Sigma^{\infty} B P, \mathbb{B} \mathcal{F}\right] \cong\left[B P, \Omega^{\infty} \mathbb{B} \mathcal{F}\right]$. The details are left to the reader.

\section{Graphs and homotopy colimits}

\subsection{Categories of dimension 1}

A small category $\mathcal{C}$ is called 1-dimensional if its nerve is 1-dimensional. Thus, in any pair of composable morphisms in $\mathcal{C}$ at least one of them is an identity morphism. Equivalently, there is a partition $\operatorname{Obj}(\mathcal{C})=C_{1} \amalg C_{2}$ such that if $\varphi: x \rightarrow y$ is a nonidentity morphism in $\mathcal{C}$ then $x \in C_{1}$ and $y \in C_{2}$.

Since there are no non-trivial compositions in $\mathcal{C}$, it is clear that if $\mathcal{D}$ is any category then any assignment $F: \operatorname{Obj}(\mathcal{C}) \rightarrow \operatorname{Obj}(\mathcal{D})$ together with an assignment of a morphism $F\left(c_{1}\right) \rightarrow F\left(c_{2}\right)$ to any non-identity morphism $c_{1} \rightarrow c_{2}$ in $\mathcal{C}$, gives rise to a functor $F: \mathcal{C} \rightarrow \mathcal{D}$.

Proposition 3.1. Let $\mathcal{C}$ be a 1-dimensional category and let $F, F^{\prime}: \mathcal{C} \rightarrow$ Top be functors whose values are $C W$-complexes. Assume that there is an isomorphism $q: F \stackrel{\simeq}{\rightarrow}$ $F^{\prime}$ in the homotopy category of spaces hoTop. Then there is a homotopy equivalence $\bar{q}: \operatorname{hocolim} F \stackrel{\simeq}{\rightarrow}$ hocolim $F^{\prime}$ with the property that the squares

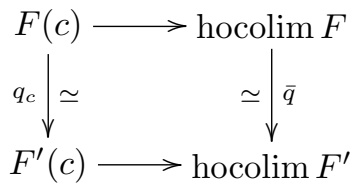

commute up to homotopy for any $c \in \mathcal{C}$.

Proof. Let $C_{1} \amalg C_{2}$ be a partition of $\operatorname{Obj}(\mathcal{C})$ as described above. The isomorphism $q: F \rightarrow F^{\prime}$ in hoTop gives rise to the following maps which we will fix once and for all. Homotopy equivalences $q_{c}: F(c) \stackrel{\simeq}{\rightarrow} F^{\prime}(c)$ for every object $c \in \mathcal{C}$ and, for every non-identity morphism $\varphi: c_{1} \rightarrow c_{2}$ in $\mathcal{C}$, a homotopy $h_{\varphi}: F\left(c_{1}\right) \times I \rightarrow F^{\prime}\left(c_{2}\right)$ from $q_{c_{2}} \circ F(\varphi)$ to $F^{\prime}(\varphi) \circ q_{c_{1}}$.

Define functors $H, H_{0}, H_{1}: \mathcal{C} \rightarrow$ Top by describing their effect on objects of $C_{1}$ and $C_{2}$ and on non-identity morphisms as follows

$$
\begin{array}{lll}
H_{0}: c_{1} \mapsto F\left(c_{1}\right), & H_{0}: c_{2} \mapsto F^{\prime}\left(c_{2}\right), & H_{0}\left(c_{1} \stackrel{\varphi}{\rightarrow} c_{2}\right)=q_{c_{2}} \circ F(\varphi) . \\
H_{1}: c_{1} \mapsto F\left(c_{1}\right), & H_{1}: c_{2} \mapsto F^{\prime}\left(c_{2}\right), & H_{1}\left(c_{1} \stackrel{\varphi}{\rightarrow} c_{2}\right)=F^{\prime}(\varphi) \circ q_{c_{1}} . \\
H: c_{1} \mapsto F\left(c_{1}\right) \times I, & H: c_{2} \mapsto F^{\prime}\left(c_{2}\right), & H\left(c_{1} \stackrel{\varphi}{\rightarrow} c_{2}\right)=h_{\varphi} .
\end{array}
$$


Now we will define natural transformations, all of which are homotopy equivalences:

$$
F \stackrel{\left(\mathrm{id}, q_{2}\right)}{\simeq} H_{0} \stackrel{\left(i_{0}, \mathrm{id}\right)}{\simeq} H \stackrel{\left(i_{1}, \mathrm{id}\right)}{\simeq} H_{1} \stackrel{\left(q_{1}, \mathrm{id}\right)}{\simeq} F^{\prime} .
$$

On objects of $C_{1}$ and $C_{2}$ the effect of these natural transformations is described by the rows of the diagram below and the columns show the effect of $F, H_{0}, H, H_{1}$ and $F^{\prime}$ on a non-identity morphism $\varphi$. The commutativity of all the squares shows that these are indeed natural transformations.

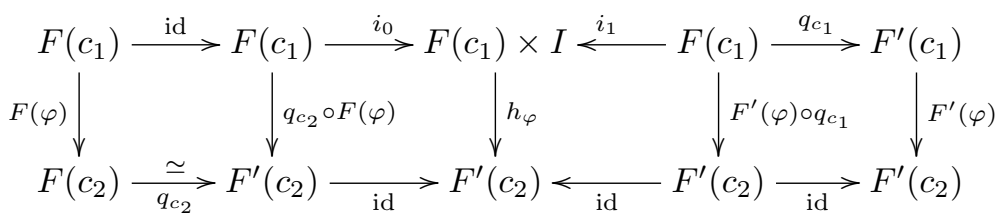

We now obtain homotopy equivalences

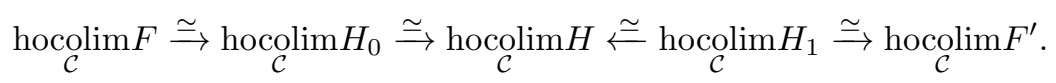

It is also clear from the commutative ladder above that the second diagram in the statement of this proposition is homotopy commutative for all $c \in \mathcal{C}$.

\subsection{Graphs and their associated categories}

A special class of 1-dimensional categories is associated to graphs. Recall from Serre [18] that a graph $Y$ consists of the following data. A set $V$ of vertices also denoted $\operatorname{vert}(Y)$ and a set $E$ of (directed) edges denoted edge $(Y)$. There is a function $E \stackrel{y \mapsto(o(y), t(y))}{\longrightarrow} V \times V$ whose components are the origin and terminus of an edge $y$. It is also equipped with an involution $E \stackrel{y \mapsto \bar{y}}{\longrightarrow} E$ which has no fixed points and "reverses the direction" of an edge, namely $o(\bar{y})=t(y)$ and $t(\bar{y})=o(y)$. The orbits of this involution $[y]$ are called "geometric edges". The set of the geometric edges of $Y$ is denoted $[\operatorname{edge}(Y)]$.

A walk in $Y$ from a vertex $u$ to a vertex $v$ is a sequence $y_{1}, \ldots, y_{n}$ of edges such that $o\left(y_{1}\right)=u$ and $t\left(y_{n}\right)=v$ and $o\left(y_{i+1}\right)=t\left(y_{i}\right)$ for all $i$. A loop is a walk from a vertex to itself. A path from $u$ to $v$ is a walk which contains no loops.

An orientation of $Y$ is a choice $A \subseteq E$ of one representative from each geometric edge. Given an orientation $A$ we will write $|y|$ for either the edge $y$ or $\bar{y}$ which belongs to $A$.

Any graph $Y$ gives rise to a small category $\mathcal{C}_{Y}$ whose object set is the disjoint union of $\operatorname{vert}(Y)$ and $[\operatorname{edge}(Y)]$. Apart from identity morphisms there is a unique morphism $[y] \stackrel{y}{\rightarrow} t(y)$ for any $y \in \operatorname{edge}(Y)$.

\subsection{Graphs of groups}

Let $Y$ be a graph. Recall from [18, Def. 8 in $\S 4.4]$ that a graph of groups $(G, Y)$ is an assignment of groups $G_{v}$ to every $v \in \operatorname{vert}(Y)$ and $G_{y}$ to every $y \in \operatorname{edge}(Y)$ such that $G_{y}=G_{\bar{y}}$ and every edge is associated with a group monomorphism $G_{y} \rightarrow G_{t(y)}$ which is denoted $a \mapsto a^{y}$ (here $a \in G_{y}$ and $a^{y}$ is its image in $G_{t(y)}$, [18, pp. 41-42]). 
We obtain an associated functor $\mathcal{G}: \mathcal{C}_{Y} \rightarrow$ Groups defined by

$$
\mathcal{G}(v)=G_{v}, \quad \mathcal{G}([y])=G_{y}, \quad \mathcal{G}([y] \stackrel{y}{\rightarrow} t(y))=G_{y} \stackrel{a \mapsto a^{y}}{\longrightarrow} G_{t(y)} .
$$

Clearly, $\mathcal{G}$ carries morphisms in $\mathcal{C}_{Y}$ to group monomorphisms. Conversely, any functor $\mathcal{G}: \mathcal{C}_{Y} \rightarrow$ Groups with this property defines a graph or groups $(G, Y)$.

\subsection{The fundamental group}

Fix a graph of groups $(G, Y)$. Let $F($ edge $(Y))$ denote the free group whose generating set is edge $(Y)$ and set

$$
\tilde{F}(G, Y)=F(\operatorname{edge}(Y)) *\left(\underset{v \in \operatorname{vert} Y}{*} G_{v}\right) .
$$

Define $F(G, Y)$ as the quotient group

$$
F(G, Y)=\tilde{F}(G, Y) /\left\langle\bar{y}=y^{-1}, y a^{y} y^{-1}=a^{\bar{y}}\right\rangle
$$

for all $y \in \operatorname{edge}(Y)$ and all $a \in G_{y}=G_{\bar{y}}$. Let $T$ be a maximal tree in $Y$. The fundamental group of $(G, Y)$ is defined as the factor group, see [18, p. 42]

$$
\pi_{1}(G, Y, T)=F(G, Y) /\langle y=1: y \in T\rangle .
$$

The image of $y$ in this group is denoted $g_{y}$. It is shown in $[\mathbf{1 8}$, p. 44] that different choices of $T$ yield isomorphic groups. By [18, Cor. 1, p. 45], the inclusions $G_{v} \leqslant$ $F(G, Y)$, for any $v \in \operatorname{vert}(Y)$, induce injections

$$
G_{v} \hookrightarrow \pi_{1}(G, Y, T)
$$

It also follows from the definitions that for any $y \in \operatorname{edge} Y$ and for any $a \in G_{y}$

$$
g_{y} \cdot a^{y} \cdot g_{y}{ }^{-1}=a^{\bar{y}} .
$$

Thus $g_{y}$ conjugates the image of $G_{y} \stackrel{a \mapsto a^{y}}{\longrightarrow} G_{t(y)}$ onto the image of $G_{y} \stackrel{a \mapsto a^{\bar{y}}}{\longrightarrow} G_{o(y)}$.

\subsection{Homotopy colimits and graphs of groups}

Composing the classifying-space functor $B(-)$ with the functor $\mathcal{G}: \mathcal{C}_{Y} \rightarrow$ Groups associated to $(G, Y)$ yields a functor $B \mathcal{G}: \mathcal{C}_{Y} \rightarrow$ Top. The result below is an observation of Dror-Farjoun in $[\mathbf{7}, \S 4.1]$.

Proposition 3.2. Consider $\mathcal{G}: \mathcal{C}_{Y} \rightarrow$ Groups associated with $(G, Y)$. Then

$$
\operatorname{hocolim}_{\mathcal{C}_{Y}} B \mathcal{G} \simeq B \pi,
$$

where $\pi=\pi_{1}(G, Y, T)$ for some choice of a maximal tree $T$ and the maps $B G_{v} \rightarrow$ hocolim $_{\mathcal{C}_{Y}} B \mathcal{G} \simeq B \pi$ are homotopic to $B\left(\right.$ incl $\left._{G_{v}}^{\pi}\right)$.

Proposition 3.3. Let $(G, Y)$ be a graph of groups and suppose that

(i) The groups $G_{v}$ and $G_{y}$ contain Sylow p-subgroups $P_{v}$ and $P_{y}$ for every $v \in \operatorname{vert} Y$ and every $y \in$ edge $Y$.

(ii) There exists a vertex $v_{0}$ such that for any other vertex $u$ of $Y$ there exists a path (directed, without loops) $y_{1}, \ldots, y_{n}$ from $v_{0}$ to $u$ such that for any $i$ the map $G_{y_{i}} \stackrel{a \mapsto a^{y_{i}}}{\longrightarrow} G_{t\left(y_{i}\right)}$ carries $P_{y_{i}}$ onto a Sylow p-subgroup of $G_{t\left(y_{i}\right)}$. 
Set $\pi=\pi_{1}(G, Y, T)$ and set $S=P_{v_{0}}$. Then hocolim $_{\mathcal{C}_{Y}} B \mathcal{G} \simeq B \pi$ and

(1) $S$ is a Sylow p-subgroup of $\pi$.

(2) If the groups $G_{v}$ are finite and if $Y$ is a tree then

(a) $B \pi$ is a p-good space $([\boldsymbol{6}, I . \S 5])$, and

(b) In the kernel $K$ of $H^{*}\left(B \pi ; \mathbb{F}_{p}\right) \stackrel{B i^{*}}{\longrightarrow} H^{*}\left(B S ; \mathbb{F}_{p}\right)$ the product of any two elements is zero.

Proof. (1) By $[\mathbf{1 8}$, I. $\S 5.3]$ the group $\pi$ acts without inversions on a tree $\tilde{X}$ such that the isotropy groups of the vertices of $\tilde{X}$ are conjugate to the subgroup $G_{v}$ of $\pi$, where $v \in \operatorname{vert} Y$. If $P$ is a finite $p$-subgroup of $\pi$ then by [18, Prop. $19, \S$ I.4.3] it fixes a vertex of $\tilde{X}$ and it is therefore conjugate in $\pi$ to a subgroup of $G_{v}$ for some vertex $v$. We therefore assume that $P \leqslant G_{v}$.

By hypothesis there is a path $y_{1}, \ldots, y_{n}$ from $v_{0}$ to $v$ with the properties listed in (ii). We will use induction on $n$ to prove that $P$ is conjugate to a subgroup of $S$. If $n=0$ then $P \leqslant G_{v_{0}}$ and the result holds because $S$ is a Sylow $p$-subgroup of $G_{v_{0}}$. Suppose that $n>0$ and note that by hypothesis the image of $G_{y_{n}}$ in $G_{t\left(y_{n}\right)}=G_{v}$, which we denote by $G_{y_{n}}^{y_{n}}$, contains a Sylow $p$-subgroup. Thus, by conjugating $P$ inside $G_{v}$ we may assume that $P \leqslant G_{y_{n}}^{y_{n}}$. As we have seen in $\S 3.4$ the element $g_{y_{n}} \in \pi$ conjugates $G_{y_{n}}^{y_{n}}$ onto $G_{y_{n}}^{\overline{y_{n}}} \leqslant G_{o\left(y_{n}\right)}=G_{t\left(y_{n-1}\right)}$. We deduce that $P$ is conjugate in $\pi$ to a subgroup of $G_{t\left(y_{n-1}\right)}$ and we can now apply the induction hypothesis to the path $y_{1}, \ldots, y_{n-1}$ and deduce that $P$ is conjugate in $\pi$ to a subgroup of $S$.

(2) Orient the tree $Y$ in the only way such that there is a path from $v_{0}$ to any vertex of $Y$ which only uses edges from the orientation class $A$. We now claim that by possibly replacing $(G, Y)$ with an isomorphic graph, the groups $P_{v}$ and $P_{y}$ can be chosen such that $(P, Y)$ is a sub-graph of $(G, Y)$, namely $G_{y} \stackrel{a \mapsto a^{y}}{\longrightarrow} G_{t(y)}$ carries $P_{y}$ into $P_{t(y)}$. In fact, by hypothesis (ii) $P_{y} \cong P_{t(y)}$ if $y \in A$.

To prove this, consider the poset $\mathcal{T}$ of the subtrees $Y^{\prime}$ of $Y$ with root $v_{0}$ for which it is possible to replace $(G, Y)$ with an isomorphic tree of groups and to find Sylow $p$-subgroup $P_{v} \leqslant G_{v}$ and $P_{y} \leqslant G_{y}$ such that $\left(P, Y^{\prime}\right)$ is a subtree of $\left(G, Y^{\prime}\right)$. First, $\mathcal{T}$ is not empty because clearly $\left\{v_{0}\right\} \in \mathcal{T}$. Next, consider a maximal element $Y^{\prime} \in \mathcal{T}$ and assume that $Y^{\prime} \neq Y$. Thus, we may assume, possibly by replacing $(G, Y)$ with an isomorphic tree, that $\left(P, Y^{\prime}\right)$ is a subtree of $\left(G, Y^{\prime}\right)$. Choose an edge $y^{\prime} \in A$ such that $v^{\prime}=o\left(y^{\prime}\right) \in Y^{\prime}$ and $v=t\left(y^{\prime}\right) \notin Y^{\prime}$.

Let $G_{\overline{y^{\prime}}}^{\overline{y^{\prime}}}$ denote the image of $G_{y^{\prime}}$ in $G_{o\left(y^{\prime}\right)}$ and let $P_{\overline{y^{\prime}}}^{\overline{y^{\prime}}}$ be the image of $P_{y^{\prime}}$. Then $g P_{\bar{y}^{\prime}}^{\overline{y^{\prime}}} g^{-1} \leqslant P_{o\left(y^{\prime}\right)}$ for some $g \in G_{o\left(y^{\prime}\right)}$ by the Sylow condition. Let $c_{g}$ denote the inner automorphism $x \mapsto g x g^{-1}$. By replacing $G_{y^{\prime}}$ with $g G_{\bar{y}^{\prime}}^{\bar{y}^{\prime}} g^{-1}$ we obtain an isomorph $(\tilde{G}, Y)$ of $(G, Y)$. Now replace $P_{y^{\prime}}$ with $g P_{\bar{y}^{\prime}}^{\bar{y}^{\prime}} g^{-1}$ and replace $P_{t\left(y^{\prime}\right)}$ with the image of $P_{y^{\prime}}$ in $G_{t\left(y^{\prime}\right)}=G_{v}$ which is a Sylow $p$-subgroup by hypothesis (ii). Note that $o\left(y^{\prime}\right) \neq t\left(y^{\prime}\right)$ so we have left the trees $\left(P, Y^{\prime}\right)$ and $\left(G, Y^{\prime}\right)$ unchanged. By construction $\left(P, Y^{\prime} \cup\{v\}\right)$ is a subtree of $\left(\tilde{G}, Y^{\prime} \cup\{v\}\right)$ which contradicts the maximality of $Y^{\prime}$. Thus, $Y \in \mathcal{T}$ which is what we wanted to prove.

(2a) Since $Y$ is a tree, $\pi$ is the amalgamated product of finite groups which is generated by the groups $G_{v}$. Also notice that the images of $P_{v}$ in $\pi$ are subgroups of $S$ because $P_{y} \rightarrow P_{t}(y)$ are isomorphisms for $y \in A$ (consider all the rooted subtrees 
$Y^{\prime} \subseteq Y$ for which $P_{v} \leqslant S$ in the amalgam, and argue as above). Let $K$ be the normal subgroup of $\pi$ generated by the elements of order prime to $p$. Clearly $K$ contains $O^{p}\left(G_{v}\right)$ and therefore together with the groups $P_{v}$ it generates $\pi$. Hence $\pi=K S$, so $\pi / K$ is a finite $p$-group.

Now $B K$ is $p$-good by [6, Proposition VII.3.2] since $K$ is $p$-perfect [6, VII.3.2] because its abelianisation must be a torsion group with no elements of order $p$. The Bousfield-Kan spectral sequence $E_{i j}^{2}=\lim _{i} H_{j}\left(B \mathcal{G} ; \mathbb{F}_{p}\right) \Rightarrow H_{i+j}\left(B \pi ; \mathbb{F}_{p}\right)$, where $\mathcal{G}$ is the diagram of groups associated to $(G, \vec{Y})$, clearly consists of finitely generated $\mathbb{F}_{p^{-}}$ modules and therefore $H_{*}\left(B \pi ; \mathbb{F}_{p}\right)$ is finite in every dimension. The action of $\pi / K$ on $H^{*}\left(B K ; \mathbb{F}_{p}\right)$ must be nilpotent by [6, II.5.2] so by the strong convergence of the Eilenberg-Moore spectral sequence for the fibration $B K \rightarrow B \pi \rightarrow B \pi / K$ proven by Dwyer in $[\mathbf{8}]$, we deduce that $H_{*}\left(B K ; \mathbb{F}_{p}\right)$ is finite in every dimension. By $[\mathbf{6}$, Chapter II, 5.1 and 5.2] we obtain a nilpotent fibration $B K_{p}^{\wedge} \rightarrow B \pi_{p}^{\wedge} \rightarrow B \pi / K$ in which both the base and the fibre are $p$-complete, hence so is the total space. This shows that $B \pi$ is $p$-good.

(2b) Let $\mathcal{G}, \mathcal{P}: \mathcal{C}_{Y} \rightarrow$ Groups be the associated functors for $(G, Y)$ and $(P, Y)$. Observe that hocolim $\mathcal{C}_{Y} B \mathcal{P}=B S$ because $P_{y} \rightarrow P_{t(y)}$ are isomorphisms for all $y \in A$ (consider the poset $\mathcal{T}$ of rooted subtrees $Y^{\prime} \subseteq Y$ for which hocolim $\left.B \mathcal{P}\right|_{\mathcal{C}_{Y^{\prime}}} \simeq B S$ and argue as above). We obtain a natural transformation of functors $B \mathcal{P} \rightarrow B \mathcal{G}$ which gives rise to a morphism of Bousfield-Kan spectral sequences $E_{2}^{*, *}(\mathcal{G}) \rightarrow E_{2}^{*, *}(\mathcal{P})$, where

$$
\begin{aligned}
& E_{2}^{i, j}(\mathcal{P})={\underset{\mathcal{C}_{Y}}{\lim _{Y}}}^{i} H^{j}\left(B \mathcal{P} ; \mathbb{F}_{p}\right) \Rightarrow H^{i+j}\left(B S ; \mathbb{F}_{p}\right), \\
& \left.E_{2}^{i, j}(\mathcal{G})={\underset{\mathcal{C}_{Y}}{\lim _{Y}}}^{i} H^{j}\left(B \mathcal{G} ; \mathbb{F}_{p}\right) \Rightarrow H^{i+j}\left(B \pi ; \mathbb{F}_{p}\right)\right) .
\end{aligned}
$$

Since $P_{v} \leqslant G_{v}$ and $P_{y} \leqslant G_{y}$ are Sylow $p$-subgroups and $G_{v}$ are finite groups, the maps $H^{*}\left(G_{v} ; \mathbb{F}_{p}\right) \rightarrow H^{*}\left(P_{v} ; \mathbb{F}_{p}\right)$ and $H^{*}\left(G_{y} ; \mathbb{F}_{p}\right) \rightarrow H^{*}\left(P_{y} ; \mathbb{F}_{p}\right)$ are injective. Since $\lim ^{0}$ is left exact, $E_{2}^{0, *}(\mathcal{G}) \rightarrow E_{2}^{0, *}(\mathcal{P})$ is injective. Also $E_{2}^{i, *}(\mathcal{P})=E_{2}^{i, *}(\mathcal{G})=0$ for all $i \geqslant 2$ because $\mathcal{C}_{Y}$ is 1-dimensional. Therefore $E_{\infty}^{* *}=E_{2}^{* *}$. We obtain the following diagram with exact rows from which it is clear that $K=\operatorname{Ker}\left(B i^{*}\right)$ is contained in $E_{\infty}^{1, *}(\mathcal{G})$.

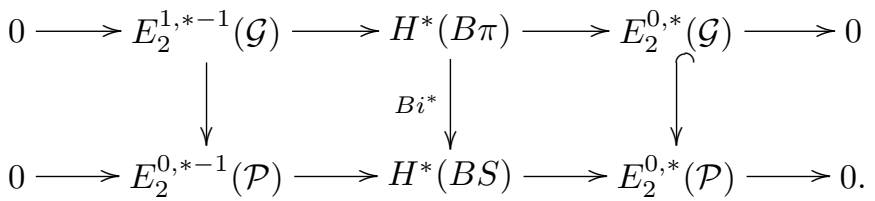

It follows that $x y=0$ for any $x, y \in K$ because $x y \in E_{\infty}^{2, *}(\mathcal{G})=0$.

\section{Groups inducing fusion systems}

\subsection{Robinson's construction - the normalizer decomposition}

Fix a $p$-local finite group $(S, \mathcal{F}, \mathcal{L})$ and a collection $\mathcal{R}$ of $\mathcal{F}$-centric subgroups which contains all the $\mathcal{F}$-centric $\mathcal{F}$-radical subgroups (e.g., all the $\mathcal{F}$-centric subgroups of $S$ ). Consider the normalizer decomposition $\delta: \bar{S}(\mathcal{R}) \rightarrow$ Top, see $\S 2.3$, and recall that in the homotopy category of spaces, $\delta$ is isomorphic to the functor $\left[R_{\bullet}\right] \mapsto B \operatorname{Aut}_{\mathcal{L}}\left(R_{\bullet}\right)$. 
Fix fully normalized representatives $R_{0}, R_{1}, \ldots, R_{n}$ for the $\mathcal{F}$-conjugacy classes of $\mathcal{R}$ and choose for convenience $R_{0}=S$. Let $\mathcal{D}$ be the subposet of $\bar{S}(\mathcal{R})$ generated by the objects $\left[R_{0}\right],\left[R_{1}\right], \ldots,\left[R_{n}\right]$ and $\left[R_{1}<S\right], \ldots,\left[R_{n}<S\right]$. It is easy to check that $\mathcal{D}=\mathcal{C}_{Y}$, where $Y$ is the tree of height 1 with root $[S]$ and edges $\left[R_{i}<S\right]$ to the leaves $\left[R_{i}\right](i=1, \ldots n)$. We obtain a tree of groups $(G, Y)$, see $\S 2.6$

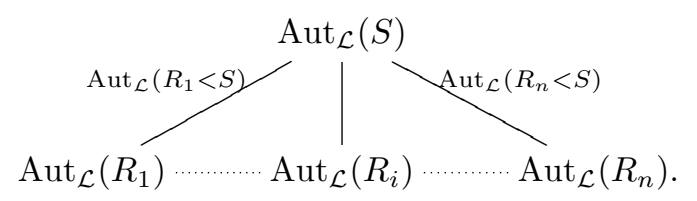

This tree of groups satisfies the conditions of Proposition 3.3 because the groups $R_{i}$ are fully normalized so $N_{S}\left(R_{i}\right)$ is a Sylow $p$-subgroup of both $\operatorname{Aut}_{\mathcal{L}}\left(R_{i}\right)$ and $\operatorname{Aut}_{\mathcal{L}}\left(R_{i}<S\right)=N_{\operatorname{Aut}_{\mathcal{L}}(S)}\left(R_{i}\right)$. Also note that $S$ is a Sylow $p$-subgroup of $\operatorname{Aut}_{\mathcal{L}}(S)$. Therefore hocolim $B \mathcal{G} \simeq B \pi_{R}$ for a group $\pi_{R}$ which is the amalgamated product of the tree (2) and which contains $S$ as a Sylow $p$-subgroup.

The crux, now, is that $B \mathcal{G}$ is the functor $\left[R_{\bullet}\right] \mapsto B \operatorname{Aut}_{\mathcal{L}}\left(R_{\bullet}\right)$ and therefore it is isomorphic to $\left.\delta\right|_{\mathcal{D}}$ in hoTop. Proposition 3.1 now yields a composite map $f$

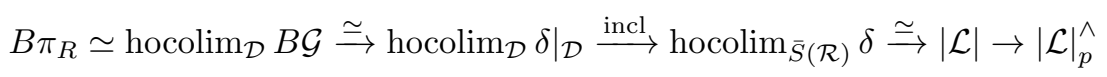

whose restriction to $B S$ is homotopic to $\theta$ in display (1). We now claim that $\mathcal{F} \subseteq$ $\mathcal{F}_{S}\left(\pi_{R}\right)$.

Proof of claim. First, $\mathcal{F}_{S}\left(\pi_{R}\right)$ contains all the groups $\operatorname{Aut}_{\mathcal{F}}\left(R_{i}\right)$ because $\pi_{R}$ contains $\operatorname{Aut}_{\mathcal{L}}\left(R_{i}\right)$ for all $i=0, \ldots, n$. Therefore $\mathcal{F}_{S}\left(\pi_{R}\right)$ contains the fusion system $\mathcal{F}^{\prime}$ on $S$ generated by $\operatorname{Aut}_{\mathcal{F}}\left(R_{i}\right)$. Clearly $\mathcal{F}^{\prime} \subseteq \mathcal{F}$ and we next claim that, in fact $\mathcal{F}^{\prime}=\mathcal{F}$. To do this, we will prove by induction on $|S: P|$ that if $\varphi \in \mathcal{F}(P, S)$ then $\varphi \in \mathcal{F}^{\prime}(P, S)$. If $|S: P|=1$ then $P=S=R_{0}$ and therefore $\varphi \in \operatorname{Aut}_{\mathcal{F}}\left(R_{0}\right) \subseteq \mathcal{F}^{\prime}$.

Assume that $|S: P|>1$. If $P$ is not $\mathcal{F}$-centric or not $\mathcal{F}$-radical then by Alperin's fusion theorem [4, Theorem A.10] it is a composition of restriction of automorphisms of $\mathcal{F}$-centric $\mathcal{F}$-radical subgroups whose order must be strictly bigger than the order of $P$ and therefore, by the induction hypothesis, all these automorphisms must belong to $\mathcal{F}^{\prime}$. Hence $\varphi \in \mathcal{F}$.

If $P$ is $\mathcal{F}$-centric $\mathcal{F}$-radical then it is $\mathcal{F}$-conjugate to $R_{i}$ for some $i$. We now claim that $P$ is $\mathcal{F}^{\prime}$-conjugate to $R_{i}$. Indeed, fix some $\mathcal{F}$-isomorphism $\alpha: R_{i} \rightarrow P$. By Alperin's fusion theorem $\alpha$ is the composition of restrictions of $\mathcal{F}$-automorphisms $\beta_{1}, \ldots, \beta_{k}$ of some $\mathcal{F}$-centric subgroups $Q_{1}, \ldots, Q_{k}$, thus $P=\beta_{k}\left(\ldots\left(\beta_{1}\left(R_{i}\right)\right) \ldots\right)$. If $\left|Q_{j}\right|=|P|$ for some $j$ then $\beta_{j}$ does not move $\beta_{j-1}\left(\ldots \beta_{1}\left(R_{i}\right) \ldots\right)$ and by omitting it we obtain another $\mathcal{F}$-isomorphism $R_{i} \rightarrow P$. Thus, by possibly replacing $\alpha$ with another $\mathcal{F}$-isomorphism, we may assume that $\left|Q_{1}\right|, \ldots,\left|Q_{k}\right|>|P|$, hence by induction hypothesis $\beta_{1}, \ldots, \beta_{k} \in \mathcal{F}^{\prime}$ and therefore $\alpha \in \mathcal{F}^{\prime}\left(R_{i}, P\right)$.

Finally, consider $\varphi \in \mathcal{F}(P, S)$, where $P$ is $\mathcal{F}$-centric $\mathcal{F}$-radical. Note that $P$ and $\varphi(P)$ are $\mathcal{F}$-conjugate to $R_{i}$ for some $i$. Choose $\mathcal{F}^{\prime}$-isomorphisms $\alpha^{\prime} \in \mathcal{F}^{\prime}\left(R_{i}, P\right)$ and $\beta^{\prime} \in \mathcal{F}^{\prime}\left(\varphi(P), R_{i}\right)$. Then $\beta^{\prime} \circ \varphi \circ \alpha^{\prime} \in \operatorname{Aut}_{\mathcal{F}}\left(R_{i}\right) \subseteq \mathcal{F}^{\prime}$ and therefore $\varphi \in \mathcal{F}^{\prime}$. This completes the induction step.

We summarize this in the following proposition. 
Proposition 4.1. Fix a p-local finite group $(S, \mathcal{F}, \mathcal{L})$. Then the group $\pi_{R}$ constructed above as the amalgamated product of (2) contains $S$ as a Sylow $p$ subgroup, it is equipped with a map $f: B \pi_{R} \rightarrow|\mathcal{L}|_{p}^{\wedge}$ whose restriction to $B S$ is homotopic to $\theta$ and furthermore, $\mathcal{F} \subseteq \mathcal{F}_{S}\left(\pi_{R}\right)$.

We can now apply Theorem 1.1(1) to $\pi_{R}$ and deduce that $\mathcal{F}=\mathcal{F}_{S}\left(\pi_{R}\right)$. Corollary 1.2 holds for $\pi_{R}$ because the implication $2 \Rightarrow 1$ is a triviality and $1 \Rightarrow 2$ follows from point (2) of Theorem 1.1.

Proposition 1.7 for $\pi_{R}$ follows from Proposition 3.3(2) because the graph of groups (2) has no loops.

Remark 4.2. The groups which appear in the amalgam (2) can be constructed directly from $\mathcal{F}$ even without the existence of an associated linking system. Thus, the group $\pi_{R}$ can be constructed for any saturated fusion system.

Indeed, since the groups $R_{i}$ are $\mathcal{F}$-centric the normalizer fusion systems $\mathcal{F}_{i}:=$ $N_{\mathcal{F}}\left(R_{i}\right)$, see $[\mathbf{4}, \S 6]$, are constrained in the sense of [2, Proposition C]. Therefore $\mathcal{F}_{i}$ admit unique linking systems $\mathcal{L}_{i}$ and there are canonically defined finite groups $L_{i}$ which contain $N_{S}\left(R_{i}\right)$ as a Sylow $p$-subgroup such that $\mathcal{F}_{i}=\mathcal{F}_{N_{S}\left(R_{i}\right)}\left(L_{i}\right)$. In fact $L_{i}=\operatorname{Aut}_{\mathcal{L}_{i}}\left(R_{i}\right)$. Similarly $\mathcal{F}_{i, 0}:=N_{\mathcal{F}}\left(R_{i}<S\right)=N_{\mathcal{F}_{0}}\left(R_{i}\right)$ are constrained fusion sub-systems of $\mathcal{F}_{0}=N_{\mathcal{F}}(S)$ and therefore they have unique linking systems $\mathcal{L}_{i, 0}$ and groups $L_{i, 0}$ such that $\mathcal{F}_{i, 0}=\mathcal{F}_{N_{S}\left(R_{i}\right)}\left(L_{i, 0}\right)$ and $L_{i, 0}=\operatorname{Aut}_{\mathcal{L}_{i, 0}}\left(R_{i}\right)$.

By construction, every morphism $P \rightarrow Q$ in $\mathcal{F}_{0}$ extends to an automorphism of $S$ and the arguments in $\S 2.6$ using the fact that $\hat{e} \in \mathcal{L}\left(R_{i}, S\right)$ is both a monomorphism and an epimorphism, show that there is a canonical isomorphism $\operatorname{Aut}_{\mathcal{L}_{0}}\left(R_{i}\right)=$ $N_{L_{0}}\left(R_{i}\right)$. Since $\mathcal{F}_{i, 0}=N_{\mathcal{F}_{0}}\left(R_{i}\right)$ the uniqueness of the linking system for $\mathcal{F}_{i, 0}$ and $[\mathbf{4}$, Definition 6.1] yield

$$
L_{i, 0}=\operatorname{Aut}_{\mathcal{L}_{i, 0}}\left(R_{i}\right)=\operatorname{Aut}_{N_{\mathcal{L}_{0}}}\left(R_{i}\right)=\operatorname{Aut}_{\mathcal{L}_{0}}\left(R_{i}\right)=N_{L_{0}}\left(R_{i}\right) \leqslant L_{0}
$$

Observe that $\mathcal{F}_{i, 0}$ is a subsystem of $\mathcal{F}_{i}$ with the same Sylow $N_{S}\left(R_{i}\right)$ which consists of the morphisms $P \rightarrow Q$ which extend to an $\mathcal{F}$-automorphism of $S$. Let $\mathcal{K}$ denote the collection of all the subgroups of $N_{S}\left(R_{i}\right)$ which contain $R_{i}$. Since both $\mathcal{F}_{i}$ and $\mathcal{F}_{i, 0}$ contain $R_{i}$ as a normal centric subgroup, $\mathcal{K}$ contains all the $\mathcal{F}_{i}$-centric-radical subgroups and all the $\mathcal{F}_{i, 0}$-centric-radical subgroups. Define a sub-category $\mathcal{L}_{i}^{\prime} \subseteq \mathcal{L}_{i}$ with object set $\mathcal{K}$ and such that $\mathcal{L}_{i}^{\prime}(P, Q)$ is the preimage of $\mathcal{F}_{i, 0}(P, Q)$ under the projection $\mathcal{L}_{i}(P, Q) \rightarrow \mathcal{F}_{i}(P, Q)$. It is clear that $\mathcal{L}_{i}^{\prime}$ is a "partial" linking system for $\mathcal{F}_{i, 0}$ in the sense that it is only defined on the subgroups in $\mathcal{K}$, but satisfies the axioms for a linking system. Our argument now follows [3, Step 7 of Theorem 4.6].

There is a functor $\mathcal{Z}: \mathcal{O}\left(\mathcal{F}_{i, 0}\right)^{\text {op }} \rightarrow \mathbf{A b}$ defined by $P \mapsto Z(P)$. By [4, Proposition 3.1], elements of $\left.\lim ^{3} \mathcal{Z}\right|_{\mathcal{K}}$ classify, up to isomorphism, the partial linking systems associated to $\mathcal{F}_{i, 0}$ defined on $\mathcal{K}$. Since $\mathcal{K}$ is closed to over-groups and contains all the $\mathcal{F}_{i, 0}$-centric-radicals, then [4, Proposition 3.2] and well known properties of the $\Lambda$-functors imply that

$$
\left.\lim _{\mathcal{O}\left(\mathcal{F}_{i, 0}^{c}\right)}{ }^{3} \mathcal{Z} \cong \underset{\mathcal{O}\left(\mathcal{F}_{i, 0}^{\mathcal{K}}\right)}{\lim ^{3}} \mathcal{Z}\right|_{\mathcal{K}}
$$

is an isomorphism. By [2, Proposition 4.2] the left hand side vanishes. This shows 
that the partial linking system $\mathcal{L}_{i}^{\prime}$ must coincide with $\mathcal{L}_{i, 0}^{\mathcal{K}}$ and in particular

$$
L_{i, 0}=\operatorname{Aut}_{\mathcal{L}_{i, 0}}\left(R_{i}\right)=\operatorname{Aut}_{\mathcal{L}_{i}^{\prime}}\left(R_{i}\right) \leqslant \operatorname{Aut}_{\mathcal{L}_{i}}\left(R_{i}\right)=L_{i} .
$$

We obtain the following tree of groups $(G, Y)$ which is defined purely by $\mathcal{F}$ without any reference to an associated linking system

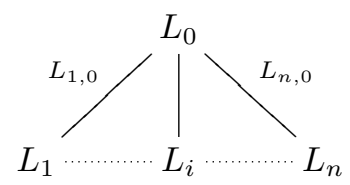

and define $\pi_{R}$ as its amalgamated product. By Proposition 3.3 it contains $S \leqslant L_{0}$ as a Sylow $p$-subgroup because $N_{S}\left(R_{i}\right)$ are Sylow $p$-subgroups of $L_{i}$ and $L_{i, 0}$. In the presence of a linking system for $\mathcal{L}$, this tree of groups coincides with (2).

We can now prove that $\mathcal{F}=\mathcal{F}_{S}\left(\pi_{R}\right)$ using our geometric methods. The assignment $\left[R_{i}\right] \mapsto \mathcal{F}_{i}$ and $\left[R_{i}<S\right] \mapsto \mathcal{F}_{i, 0}$ define a functor $\nu$ from $\mathcal{C}_{Y}$ to the category of fusion sub-systems of $\mathcal{F}$. By applying Ragnarsson's functor $\mathbb{B}$ we obtain a functor $\mathbb{B} \nu: \mathcal{C}_{Y} \rightarrow$ Spectra together with a map $\mathbb{B} \nu \rightarrow \mathbb{B} \mathcal{F}$. Since $\mathcal{F}_{i}$ and $\mathcal{F}_{i, 0}$ are constrained, $\mathbb{B} \nu \simeq \Sigma^{\infty} B \mathcal{G}_{p}^{\wedge}$ for the functor $\mathcal{G}$ associated to the tree (3). By adjunction we obtain $B \mathcal{G} \rightarrow \Omega^{\infty} \mathbb{B} \mathcal{F}$ in hoTop and by Propositions 3.1 and 3.3 we obtain $f: B \pi_{R} \rightarrow \Omega^{\infty} \mathbb{B} \mathcal{F}$ whose restriction to $B S$ is homotopic to $\sigma_{\mathcal{F}}^{\#}$. Proposition 2.2 implies that $\mathcal{F}_{S}\left(\pi_{R}\right) \subseteq \mathcal{F}$ and the argument we used above exploiting Alperin's fusion theorem shows that $\mathcal{F} \subseteq \mathcal{F}_{S}\left(\pi_{R}\right)$.

Remark 4.3. Robinson's originally constructed in $[\mathbf{1 7}]$ a group $\Gamma$ as the amalgamated product (3) but with the groups $L_{i, 0}$ replaced with their Sylow $p$-subgroup $N_{S}\left(R_{i}\right)$. The group $\pi_{R}$ that we construct is therefore a quotient of $\Gamma$. Note however that if $\pi_{R}$ admits a signaliser functor, then $\mathcal{F}$ has an associated linking system and we can apply Theorem 1.1 to $B \Gamma \rightarrow B \pi_{R} \rightarrow|\mathcal{L}|_{p}^{\wedge}$ in order to obtain a signaliser functor on $\Gamma$.

Remark 4.4. In [13, Theorem 5.5] it is shown that a $p$-local finite group with a "compressible $\mathcal{F}$-centric" collection $\mathcal{R}$ admits a group $\Gamma$ which contains $S$ as a Sylow $p$-subgroup with the property that $\mathcal{F}=\mathcal{F}_{S}(\Gamma)$ and that $\mathcal{L}$ is induced by some signaliser functor on $\Gamma$. The group $\Gamma$ is simply the group $\pi_{R}$. Our techniques are inspired by this paper.

\subsection{Leary-Stancu's construction - the subgroup decomposition}

The following argument is explicit in $[\mathbf{1 9}]$. Fix a $p$-local finite group $(S, \mathcal{F}, \mathcal{L})$ and let $\Phi=\left\{\varphi_{1}, \ldots, \varphi_{n}\right\}$ be a set of morphisms $\varphi_{i}: P_{i} \rightarrow S$ in $\mathcal{F}^{c}$ which, together with $\operatorname{Inn}(S)$, generate $\mathcal{F}$ (recall Alperin's fusion theorem). For example, $\Phi$ could be the set of all the morphisms in $\mathcal{F}^{c}$.

Consider the oriented graph $Y$ with just one vertex $v_{0}$ and oriented edges $y_{1}, \ldots, y_{n}$. Define a graph of group $(G, Y)$, where $G_{v_{0}}=S, G_{y_{i}}=G_{\overline{y_{i}}}=P_{i}$ and $y_{i} \mapsto P_{i} \stackrel{\varphi_{i}}{\longrightarrow} S$ and $\overline{y_{i}} \mapsto P_{i} \stackrel{\text { incl }}{\longrightarrow} S$. Thus, the functor $\mathcal{G}: \mathcal{C}_{Y} \rightarrow$ Groups is depicted by

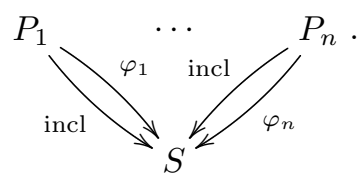


Define a functor $J: \mathcal{C}_{Y} \rightarrow \mathcal{O}\left(\mathcal{F}^{c}\right)$ by

$$
J\left(v_{0}\right)=S, \quad J\left(\left[y_{i}\right]\right)=P_{i}, \quad J\left(\bar{y}_{i}\right)=P_{i} \stackrel{\text { incl }}{\longrightarrow} S, \quad J\left(y_{i}\right)=P_{i} \stackrel{\varphi_{i}}{\longrightarrow} S .
$$

Define $\pi_{L S}:=\pi_{1}(G, Y, T)$, where the maximal tree $T$ must be $\left\{v_{0}\right\}$.

Observe that $J^{*} \beta \cong B \mathcal{G}$ in hoTop where $\beta$ is the subgroup decomposition, see $\S 2.5$. Now we obtain from Proposition 3.3, whose conditions are satisfied trivially, and Proposition 3.1, and the properties of $\beta$ described in $\S 2.6$, a sequence of maps

$$
B \pi_{L S} \simeq \operatorname{hocolim}_{\mathcal{C}_{Y}} B \mathcal{G} \stackrel{\simeq}{\rightarrow} \operatorname{hocolim}_{\mathcal{C}_{Y}} J^{*} \beta \stackrel{J_{*}}{\rightarrow} \operatorname{hocolim}_{\mathcal{O}\left(\mathcal{F}^{c}\right)} \beta \stackrel{\simeq}{\rightarrow}|\mathcal{L}| \rightarrow|\mathcal{L}|_{p}^{\wedge}
$$

whose restriction to $B S$ is homotopic to $\theta$. We also observe that $\mathcal{F} \subseteq \mathcal{F}_{S}\left(\pi_{L S}\right)$ because $S \leqslant \pi_{L S}$ and by construction of $\pi_{1}(G, Y, T)$, see $\S 3.4$, every $\varphi_{i}: P \rightarrow S$ in $\Phi$ is obtained by conjugation by the element $g_{y_{i}}$. To summarize, we have shown:

Proposition 4.5. Fix a p-local finite group $(S, \mathcal{F}, \mathcal{L})$. Then the group $\pi_{L S}$ contains $S$ as a Sylow p-subgroup, it is equipped with a map $f: B \pi_{L S} \rightarrow|\mathcal{L}|_{p}^{\wedge}$ whose restriction to $B S$ is homotopic to $\theta$ and $\mathcal{F} \subseteq \mathcal{F}_{S}\left(\pi_{L S}\right)$.

Thus, we can apply Theorem 1.1(1) to recover the results of $[\mathbf{9}]$, namely $\mathcal{F}=$ $\mathcal{F}_{S}\left(\pi_{L S}\right)$. Also Corollary 1.2 for $\pi_{L S}$ follows since the implication $2 \Rightarrow 1$ is a triviality and $1 \Rightarrow 2$ follows from point (2) of Theorem 1.1.

Remark 4.6. The construction of $\pi_{L S}$ appears in [9]. It is clear that it is defined purely in terms of $\mathcal{F}$. The same ideas of Remark 4.2 can be used to obtain $\mathcal{F}=\mathcal{F}_{S}\left(\pi_{L S}\right)$ for any saturated fusion system without the assumption that a linking system exists.

\subsection{Aschbacher-Chermak's construction - the normalizer decomposition}

Fix an odd prime $q$ and some $n \geqslant 1$. We now recall from [10], see also [11], the construction of Solomon's fusion system $\mathcal{F}_{\text {Sol }}\left(q^{n}\right)$ at the prime 2 . This fusion system is obtained from the fusion system of $\operatorname{Spin}_{7}\left(q^{n}\right)$ as follows. Throughout we write $q^{n}$ for the finite field $\mathbb{F}_{q^{n}}$ and $q^{\infty}$ for its algebraic closure. There is a group homomorphism $\omega: \mathrm{SL}_{2}\left(q^{\infty}\right)^{3} \rightarrow \operatorname{Spin}_{7}\left(q^{\infty}\right)$ whose kernel is the central subgroup of order 2 generated by $(-I,-I,-I)$. We will denote $\omega\left(X_{1}, X_{2}, X_{3}\right)=\llbracket X_{1}, X_{2}, X_{3} \rrbracket$.

Let $H\left(q^{\infty}\right)$ be the image of $\omega$. The centre of $\operatorname{Spin}_{7}\left(q^{\infty}\right)$ is a group of order 2 generated by $z=\llbracket I, I,-I \rrbracket$. The set $\{\llbracket \pm I, \pm I, \pm I \rrbracket\}$ forms an elementary abelian group $U$ of rank 2. By [10, Lemma 2.3] there is an element $\tau \in N_{\operatorname{Spin}_{7}(q)}(U)$ such that $c_{\tau}\left(\llbracket X_{1}, X_{2}, X_{3} \rrbracket\right)=\llbracket X_{2}, X_{1}, X_{3} \rrbracket$, where $c_{\tau}$ denotes conjugation $g \mapsto \tau g \tau^{-1}$. Note that $\tau$ normalizes $H\left(q^{\infty}\right)$.

Fix a copy of the quaternion group $Q_{8}$ in $\mathrm{SL}_{2}(q)$ and choose generators $A, B$ of order 4 . Set (cf. $[\mathbf{1 1}, \S 1])$

$$
\begin{aligned}
C\left(q^{\infty}\right) & =\left\{X \in C_{\mathrm{SL}_{2}\left(q^{\infty}\right)}(A): X^{2^{k}}=I \text { for some } k\right\} \cong \mathbb{Z} / 2^{\infty} \\
Q\left(q^{\infty}\right) & =\left\langle C\left(q^{\infty}\right), B\right\rangle \\
S_{0}\left(q^{\infty}\right) & =\omega\left(Q\left(q^{\infty}\right)^{3}\right) \leqslant H\left(q^{\infty}\right) \\
S\left(q^{\infty}\right) & =S_{0}\left(q^{\infty}\right) \cdot\langle\tau\rangle .
\end{aligned}
$$


We will write $\Theta\left(q^{n}\right)=\Theta\left(q^{\infty}\right) \cap \operatorname{Spin}_{7}\left(q^{n}\right)$, where $\Theta$ denotes one of $C(-), Q(-)$, $S_{0}(-)$, or $S(-)$, etc. By [10, Lemma 2.7], $S\left(q^{n}\right)$ is a Sylow 2-subgroup of $\operatorname{Spin}_{7}\left(q^{n}\right)$ hence also of $H\left(q^{n}\right) \cdot\langle\tau\rangle$. Also $S_{0}\left(q^{n}\right)$ is a Sylow 2-subgroup of $H\left(q^{n}\right)$.

There are automorphisms $\hat{\gamma}$ and $\hat{\delta}_{u}$ of $S_{0}\left(q^{\infty}\right)$ defined in [11, Definition 1.6] by

$$
\hat{\gamma}\left(\llbracket X_{1}, X_{2}, X_{3} \rrbracket\right)=\llbracket X_{3}, X_{1}, X_{2} \rrbracket, \quad \hat{\delta}_{u}\left(\llbracket X_{1}, X_{2}, A^{\prime} B^{j} \rrbracket\right)=\llbracket X_{1}, X_{2},\left(A^{\prime}\right)^{u} B^{j} \rrbracket,
$$

where $A^{\prime} \in C\left(q^{\infty}\right)$ and $X_{1}, X_{2}, X_{3} \in Q\left(q^{\infty}\right)$ and $u$ is a carefully chosen 2-adic unit. Set

$$
\Gamma_{n}=\left\langle\operatorname{Inn}\left(S_{0}\left(q^{n}\right)\right), c_{\tau}, \hat{\delta}_{u} \circ \hat{\gamma} \circ \hat{\delta}_{u}^{-1}\right\rangle \leqslant \operatorname{Aut}\left(S_{0}\left(q^{n}\right)\right) .
$$

The fusion system $\mathcal{F}_{\text {Sol }}\left(q^{n}\right)$ on $S=S\left(q^{n}\right)$ is the one generated by $\mathcal{F}_{S}\left(\operatorname{Spin}_{7}\left(q^{n}\right)\right)$ and by $\mathcal{F}_{S_{0}\left(q^{n}\right)}\left(\Gamma_{n}\right)$.

Set $S=S\left(q^{n}\right)$ and $S_{0}=S_{0}\left(q^{n}\right)$. It easily follows from [10, Proposition 2.5] and the definitions that $C_{S}(U)=S_{0}$. Also, $\left|S: S_{0}\right|=2$ because $\left|S\left(q^{\infty}\right): S_{0}\left(q^{\infty}\right)\right|=2$ and therefore $S_{0}$ is fully normalized. It is also $\mathcal{F}$-centric by the next proposition because by construction of $\mathcal{F}$, the $\mathcal{F}$-conjugates of $S_{0} \operatorname{are~}_{\operatorname{Spin}_{7}}\left(q^{n}\right)$-conjugates.

Proposition 4.7. $C_{\operatorname{Spin}_{7}\left(q^{n}\right)}\left(S_{0}\left(q^{n}\right)\right) \subseteq S_{0}\left(q^{n}\right)$.

Proof. Since $U \subseteq S_{0}$ we deduce from [10, Proposition 2.5] that $C_{\operatorname{Spin}_{7}\left(q^{n}\right)}\left(S_{0}\right) \subseteq$ $C_{\operatorname{Spin}_{7}\left(q^{n}\right)}(U)=H\left(q^{n}\right)$ which contains $S_{0}$ as a Sylow 2-subgroup. It follows that $C_{\operatorname{Spin}_{7}\left(q^{n}\right)}\left(S_{0}\right)=Z\left(S_{0}\right) \times C^{\prime}$, where $C^{\prime}$ has odd order. We need to show that $C^{\prime}=1$. Choose $x \in C^{\prime}$ of order $r$. Since Ker $\omega$ is central of order 2 then $\omega^{-1}(x)$ consists of an element $\tilde{x}_{1}$ of order $r$ and an element $\tilde{x_{2}}$ of order $2 r$. Now $\left(Q_{8}\right)^{3} \subseteq \omega^{-1}\left(S_{0}\right)$ must leave $\left\{\tilde{x}_{1}, \tilde{x}_{2}\right\}$ invariant because $S_{0}$ centralizes $x$ and since the orders of $\tilde{x}_{1}, \tilde{x}_{2}$ are different, $\left(Q_{8}\right)^{3}$ centralizes these elements. By Lemma 4.8 below $\tilde{x}_{1} \in Q_{8}$ and therefore $\tilde{x}_{1}=1$ which implies that $x=1$.

Lemma 4.8. Fix an odd prime $q$. Then

(1) The centralizer of any element $X$ of order 4 in $\mathrm{SL}_{2}\left(q^{n}\right)$ is a cyclic group.

(2) Any quaternion group $Q_{8}$ in $\mathrm{SL}_{2}\left(q^{\infty}\right)$ contains its centralizer.

Proof. (1) The minimal polynomial of $X$ is $x^{2}+1$ which splits in $\mathbb{F}_{q^{n+1}}$ which contains $\zeta=\sqrt{-1}$. Thus, $X$ is conjugate in $\mathrm{GL}_{2}\left(q^{n+1}\right)$ to the diagonal matrix $\operatorname{diag}(\zeta,-\zeta)$ whose centralizer is the subgroup of diagonal matrices and therefore its $\mathrm{SL}_{2}\left(q^{n+1}\right)$ centralizer is the cyclic group $\mathbb{F}_{q^{n+1}}^{\times}$. Conjugating back we see that $C_{\mathrm{SL}_{2}\left(q^{n+1}\right)}(X)$ is cyclic hence so is $C_{\mathrm{SL}_{2}\left(q^{n}\right)}(X)$.

(2) Fix any $n$ such that $Q_{8} \leqslant \mathrm{SL}_{2}\left(q^{n}\right)$ and consider $X$ in its centralizer. Now $Q_{8}$ is generated by elements $A, B$ of order 4 , so $X$ belongs to the cyclic groups $C_{\mathrm{SL}_{2}\left(q^{n}\right)}(A)$ and $C_{\mathrm{SL}_{2}\left(q^{n}\right)}(B)$. If $\langle A\rangle \subseteq\langle X\rangle$ and $\langle B\rangle \subseteq\langle X\rangle$ then $Q_{8} \leqslant\langle X\rangle$ which is absurd. So either $\langle X\rangle \subseteq\langle A\rangle$ or $\langle X\rangle \subseteq\langle B\rangle$, hence $X \in Q_{8}$.

Set $\mathcal{F}=\mathcal{F}_{\text {Sol }}\left(q^{n}\right)$ and $\mathcal{L}=\mathcal{L}_{\text {Sol }}\left(q^{n}\right)$ and $Z$ is the subgroup generated by the element $z$ (this is the centre of $\left.\operatorname{Spin}_{7}\left(q^{n}\right)\right)$. Set $\operatorname{Aut}_{\mathcal{L}}\left(S_{0} ; Z\right)=C_{\mathrm{Aut}_{\mathcal{L}}\left(S_{0}\right)}(Z)$. By [10, Propositions $2.11,3.3]$ the linking system $\mathcal{L}_{\text {Sol }}\left(q^{n}\right)$ contains $\mathcal{L}_{S}^{c}\left(\operatorname{Spin}_{7}\left(q^{n}\right)\right)$ as a subcategory. In fact, $\mathcal{L}_{S}^{c}\left(\operatorname{Spin}_{7}\left(q^{n}\right)\right)=C_{\mathcal{L}}(Z)$ is the centralizer linking system [4, Definition 2.4] 
and by Proposition 4.7 and [4, remarks before Definiton 1.6]

$$
\begin{aligned}
\operatorname{Aut}_{\mathcal{L}}\left(S_{0} ; Z\right)=\operatorname{Aut}_{C_{\mathcal{L}}(Z)}\left(S_{0}\right)= & N_{\operatorname{Spin}_{7}\left(q^{n}\right)}\left(S_{0}\right) / C_{\operatorname{Spin}_{7}\left(q^{n}\right)}^{\prime}\left(S_{0}\right) \\
& =N_{\operatorname{Spin}_{7}\left(q^{n}\right)}\left(S_{0}\right) \leqslant \operatorname{Spin}_{7}\left(q^{n}\right) .
\end{aligned}
$$

We obtain inclusions of subcategories of $\mathcal{L}$

$$
\mathcal{L}_{S}\left(\operatorname{Spin}_{7}\left(q^{n}\right)\right) \longleftrightarrow \operatorname{BAut}_{\mathcal{L}}\left(S_{0} ; Z\right) \longrightarrow \operatorname{BAut}_{\mathcal{L}}\left(S_{0}\right) .
$$

It gives rise to the diagram of spaces below, all of whose squares commute, except the one on the top left which only commutes up to homotopy.

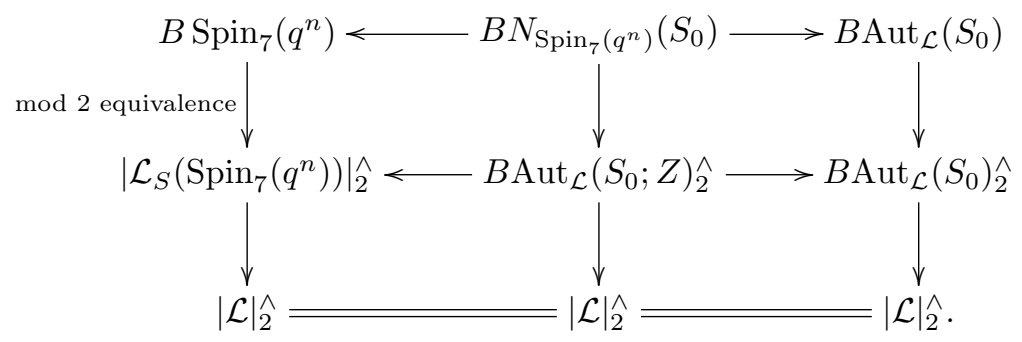

We observe that $N_{\operatorname{Spin}_{7}\left(q^{n}\right)}\left(S_{0}\right)$ contains the Sylow subgroup $S$ because $\left|S: S_{0}\right|=2$. By Proposition 3.3, the homotopy colimit of the first row is homotopy equivalent to $B \pi_{A C}$, where $\pi_{A C}$ is the amalgam of the tree

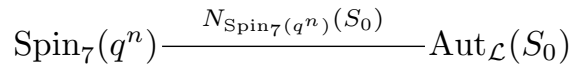

which contains $S$ as a Sylow 2-subgroup. Proposition 3.1 implies that there is a map $f: B \pi_{A C} \rightarrow|\mathcal{L}|_{2}^{\wedge}$ whose restriction to $B S$ is homotopic to $\theta$. Moreover, $\operatorname{Spin}_{7}\left(q^{n}\right)$ and $\operatorname{Aut}_{\mathcal{L}}\left(S_{0}\right)$ are subgroups of $\pi_{A C}$ and also $\operatorname{Aut}_{\mathcal{F}}\left(S_{0}\right)=\operatorname{Aut}_{\mathrm{Aut}_{\mathcal{L}}\left(S_{0}\right)}\left(S_{0}\right)$ contains $c_{\tau}$ and $\hat{\delta}_{u} \circ \hat{\gamma} \circ \hat{\delta}_{u}^{-1}$ because these homomorphisms clearly normalize $S_{0}\left(q^{n}\right)$. We therefore deduce that $\mathcal{F} \subseteq \mathcal{F}_{S}(\pi)$. Thus we have proven:

Proposition 4.9. The group $\pi_{A C}$ satisfies the hypotheses of Theorem 1.1. In particular $\mathcal{F}=\mathcal{F}_{S}\left(\pi_{A C}\right)$ and $\pi_{A C}$ has a signaliser functor which induces $\mathcal{L}$. It also satisfies the condition of Proposition 3.3(2) because (4) has no loops. This proves the statement in Proposition 1.7 regarding $\pi_{A C}$.

Remark 4.10. The group $\Gamma$ that Aschbacher and Chermak constructed in $[\mathbf{1}]$ is the amalgamated product

$$
\operatorname{Spin}_{7}\left(q^{n}\right) \frac{N_{\operatorname{Spin}_{7}\left(q^{n}\right)}(U)}{} K,
$$

where $\left|K: N_{\operatorname{Spin}_{7}\left(q^{n}\right)}(U)\right|=3$. They need the hypothesis that $q \cong 3$ or $5 \bmod 8$, however, which we don't.

Indeed, the groups $\Gamma$ and $\pi_{A C}$ are different. We will show below that $U$ is the unique normal subgroup of $S_{0}=S_{0}\left(q^{n}\right)$ that is isomorphic to the Klein group $\mathbb{Z} / 2 \oplus$ $\mathbb{Z} / 2$ and therefore $N_{\operatorname{Spin}_{7}\left(q^{n}\right)}\left(S_{0}\right) \subseteq N_{\operatorname{Spin}_{7}\left(q^{n}\right)}(U)$. The inclusion is proper because $H\left(q^{n}\right)=C_{\operatorname{Spin}_{7}\left(q^{n}\right)}(U)$ does not normalize $S_{0}\left(q^{n}\right)$. In addition, $\operatorname{Aut}_{\operatorname{Spin}_{7}\left(q^{n}\right)}(U)=C_{2}$ and $\operatorname{Aut}_{\mathcal{F}_{\text {Sol }}\left(q^{n}\right)}(U)=\Sigma_{3}$ and $\operatorname{Aut}_{\mathcal{F}_{\text {Sol }}\left(q^{n}\right)}\left(S_{0}\right)$ contains $\hat{\delta}_{u} \hat{\gamma} \hat{\delta}_{u}^{-1}$ from which we deduce 
that $\left|\operatorname{Aut}_{\mathcal{L}}\left(S_{0}\right): N_{\operatorname{Spin}_{7}\left(q^{n}\right)}\left(S_{0}\right)\right|=3$. Thus the groups we use in the amalgam (4) are smaller than those used by Aschbacher and Chermak. It is interesting to see how our approach interprets the signaliser functors geometrically in Theorem 1.1 rather than group-theoretically.

Claim. $U$ is the unique normal subgroup of $S_{0}\left(q^{n}\right)$ isomorphic to the Klein group.

Proof. Consider a Klein group $E \triangleleft S_{0}$ and set $\tilde{E}=\omega^{-1}(E)$. Note that $\tilde{E}$ is normalized by $\left(Q_{8}\right)^{3} \leqslant Q\left(q^{\infty}\right)^{3}$. Fix an element $\tilde{X}=\left(X_{1}, X_{2}, X_{3}\right) \in \tilde{E}$ and note that $X_{i}^{2}= \pm I$ because all the elements of $E$ have order 2 and $\operatorname{Ker} \omega= \pm(I, I, I)$. If $X_{i}$ has order 4 then $X_{1}^{2}=X_{2}^{2}=X_{3}^{2}=-I$ so $X_{1}, X_{2}, X_{3}$ have order 4 and Lemma 4.8 implies that $\tilde{E}$ contains at least $2^{3}$ different $\left(Q_{8}\right)^{3}$ conjugates of $\tilde{X}$. This is impossible, so the order of $\tilde{X}$ is at most 2 .

If $E \neq U$ then we may assume that $X_{1} \neq \pm I$ hence $X_{1}=W B^{ \pm 1}$ for some $W \in$ $C\left(q^{\infty}\right)$. Now it follows that $\tilde{X} \cdot B \tilde{X} B^{-1}=\left(-W^{2}, I, I\right) \in \tilde{E}$ must also have order 2 . This can only happen if $W \in\langle A\rangle \leqslant C\left(q^{\infty}\right)$ which implies that $X_{1} \in Q_{8}$ whose only element of order 2 is $-I$ and this is absurd.

Remark 4.11. The construction which we have presented here is motivated by the normalizer decomposition with respect to the collection of the elementary abelian subgroups $\mathcal{E}$, see [12]. If $\mathcal{D}$ is the subposet of $\bar{S}(\mathcal{E})$ of the form $[Z] \leftarrow[Z<U] \rightarrow[U]$ then the restriction of the normalizer decomposition to $\mathcal{D}$ has the form

$$
\left|C_{\mathcal{L}}(Z)\right| \longleftarrow\left|C_{\mathcal{L}}(U)\right|_{h \operatorname{Aut}_{\mathcal{F}}(Z<U)} \longrightarrow\left|\mathcal{C}_{\mathcal{L}}(U)\right|_{h \operatorname{Aut}_{\mathcal{F}}(U)} .
$$

Up to 2-completion, the space on the left is $B \operatorname{Spin}_{7}\left(q^{n}\right)$. A careful examination of the constructions in $[\mathbf{1 2}$, Theorem $B]$ shows that these maps are induced by inclusion of categories which contain $\mathcal{B A u t}_{C_{\mathcal{L}}(Z)}\left(S_{0}\right)$ and $\mathcal{B} \operatorname{Aut}_{\mathcal{L}}\left(S_{0}\right)$ as very natural full subcategories to look at. We omit the details.

\section{Acknowledgements}

The second author was supported by grant ANR BLAN08-2-338236.

\section{References}

[1] M. Aschbacher, A. Chermak, A group-theoretic approach to a family of 2-local finite groups constructed by Levi and Oliver. Ann. of Math. (2) 171 (2010), no. $2,881-978$.

[2] C. Broto, N. Castellana, J. Grodal, R. Levi, B. Oliver, Subgroup families controlling p-local finite groups. Proc. London Math. Soc. (3) 91 (2005), no. 2, $325-354$.

[3] C. Broto, N. Castellana, J. Grodal, R. Levi, B. Oliver, Extensions of $p$-local finite groups. Trans. Amer. Math. Soc. 359 (2007), no. 8, 3791-3858.

[4] C. Broto, R. Levi, B. Oliver, The homotopy theory of fusion systems. J. Amer. Math. Soc. 16 (2003), no. 4, 779-856.

[5] C. Broto, R. Levi, B. Oliver, The theory of $p$-local groups: a survey. Homotopy theory: relations with algebraic geometry, group cohomology, and algebraic $K$ theory, 51-84, Contemp. Math., 346, Amer. Math. Soc., Providence, RI, 2004. 
[6] A. K. Bousfield, D. M. Kan, Homotopy limits, completions and localizations. Lecture Notes in Mathematics, Vol. 304. Springer-Verlag, Berlin-New York, 1972.

[7] E. Dror Farjoun, Fundamental group of homotopy colimits. Adv. Math. 182 (2004), no. 1, 1-27.

[8] W. G. Dwyer, Strong convergence of the Eilenberg-Moore spectral sequence. Topology 13 (1974), 255-265.

[9] Leary, Ian J., Stancu, Radu. Realising fusion systems. Algebra Number Theory 1 (2007), no. $1,17-34$.

[10] R. Levi, B. Oliver, Construction of 2-local finite groups of a type studied by Solomon and Benson. Geom. Topol. 6 (2002), 917-990.

[11] R. Levi, B. Oliver, Correction to: "Construction of 2-local finite groups of a type studied by Solomon and Benson". Geom. Topol. 9 (2005), 2395-2415.

[12] A. Libman, The normaliser decomposition for $p$-local finite groups. Algebr. Geom. Topol. 6 (2006), 1267-1288.

[13] A. Libman, A. Viruel, On the homotopy type of the non-completed classifying space of a p-local finite group. Forum Math. 21 (2009), no. 4, 723-757.

[14] B. Oliver, J. Ventura, Extensions of linking systems with p-group kernel. Math. Ann. 338 (2007), no. 4, 983-1043.

[15] L. Puig, Frobenius categories. J. Algebra 303 (2006), no. 1, 309-357.

[16] K. Ragnarsson, Classifying spectra of saturated fusion systems. Algebr. Geom. Topol. 6 (2006), 195-252.

[17] G. Robinson, Amalgams, blocks, weights, fusion systems and finite simple groups. J. Algebra 314 (2007), no. 2, 912-923.

[18] Serre, Jean-Pierre, Trees. Springer-Verlag, Berlin-New York, 1980.

[19] N. Seeliger, Group models for fusion systems. Topology Appl. 159 (2012), no. $12,2845-2853$.

[20] E. H. Spanier, Algebraic topology. McGraw-Hill Book Co., New York-Toronto, Ont.-London 1966.

Assaf Libman a.libman@abdn.ac.uk

Department of Mathematical Sciences, King's College, Aberdeen AB24 3UE, Scotland, U.K.

Nora Seeliger seeliger@mpim-bonn.mpg.de

Max-Planck-Institut für Mathematik, Vivatsgasse 7, D-53111 Bonn, Germany 